\title{
Obscuration effects in super-soft-source X-ray spectra
}

\author{
J.-U. Ness ${ }^{1}$, J. P. Osborne ${ }^{2}$, M. Henze ${ }^{1}$, A. Dobrotka ${ }^{3}$, J. J. Drake 4 , V. A. R. M. Ribeiro ${ }^{5}$, S. Starrfield ${ }^{6}$, E. Kuulkers ${ }^{1}$, \\ E. Behar ${ }^{7}$, M. Hernanz ${ }^{8}$, G. Schwarz ${ }^{9}$, K. L. Page ${ }^{2}$, A. P. Beardmore ${ }^{2}$, and M. F. Bode ${ }^{10}$ \\ ${ }^{1}$ Science Operations Division, Science Operations Department of ESA, ESAC, 28691 Villanueva de la Cañada (Madrid), Spain \\ e-mail: juness@sciops.esa.int \\ 2 Department of Physics \& Astronomy, University of Leicester, Leicester, LE1 7RH, UK \\ 3 Department of Physics, Institute of Materials Science, Faculty of Materials Science and Technology, \\ Slovak University of Technology in Bratislava, Paulinska 16, 91724 Trnava, Slovak Republic \\ ${ }^{4}$ Harvard-Smithsonian Center for Astrophysics, 60 Garden Street, Cambridge, MA 02138, USA \\ 5 Astrophysics, Cosmology and Gravity Centre, Department of Astronomy, University of Cape Town, Private Bag X3, \\ 7701 Rondebosch, South Africa \\ ${ }^{6}$ School of Earth and Space Exploration, Arizona State University, Tempe, AZ 85287-1404, USA \\ 7 Physics Department, Technion, 32000 Haifa, Israel \\ 8 Institut de Ciències de l'Espai (CSIC-IEEC), Campus UAB, Facultat de Ciències, C5 parell 2 ${ }^{\text {on }}, 08193$ Bellaterra (Barcelona), \\ Spain \\ 9 American Astronomical Society, 2000 Florida Ave., NW, Suite 400, DC 20009-1231, USA \\ 10 Astrophysics Research Institute, Liverpool John Moores University, IC2 Liverpool Science Park, 146 Brownlow Hill, L3 5RF, UK
}

Received 31 July 2013 / Accepted 10 September 2013

\section{ABSTRACT}

\begin{abstract}
Context. Super-soft-source (SSS) X-ray spectra are blackbody-like spectra with effective temperatures $\sim 3-7 \times 10^{5} \mathrm{~K}$ and luminosities of $10^{35-38} \mathrm{erg} \mathrm{s}^{-1}$. Grating spectra of SSS and novae in outburst that show SSS type spectra display atmospheric absorption lines. Radiation transport atmosphere models can be used to derive physical parameters. Blue-shifted absorption lines suggest that hydrostatic equilibrium is an insufficient assumption, and more sophisticated models are required.

Aims. In this paper, we bypass the complications of spectral models and concentrate on the data in a comparative, qualitative study. We inspect all available X-ray grating SSS spectra to determine systematic, model-independent trends.

Methods. We collected all grating spectra of conventional SSS like Cal 83 and Cal 87 plus observations of novae during their SSS phase. We used comparative plots of spectra of different systems to find common and different features. The results were interpreted in the context of system parameters obtained from the literature.

Results. We find two distinct types of SSS spectra that we name SSa and SSe. Their main observational characteristics are either clearly visible absorption lines or emission lines, respectively, while both types contain atmospheric continuum emission. SSa spectra are highly structured with no spectral model currently able to reproduce all details. The emission lines clearly seen in SSe may also be present in SSa, hidden within the forest of complex atmospheric absorption and emission features. This suggests that SSe are in fact obscured SSa systems. Similarities between SSe and SSa with obscured and unobscured AGN, respectively, support this interpretation. We find all known or suspected high-inclination systems to emit permanently in an SSe state. Some sources are found to transition between SSa and SSe states, becoming SSe when fainter.

Conclusions. SSS spectra are subject to various occultation processes. In persistent SSS spectra such as Cal 87 , the accretion disc blocks the central hot source when viewed edge on. In novae during their SSS phase, the accretion disc may have been destroyed during the initial explosion but could have reformed by the time of the SSS phase. In addition, clumpy ejecta may lead to temporary obscuration events. The emission lines stem from reprocessed emission in the accretion disc, its wind or further out in clumpy ejecta, while Thomson scattering allows continuum emission to be visible also during total obscuration of the central hot source.
\end{abstract}

Key words. novae, cataclysmic variables - X-rays: binaries - binaries: eclipsing

\section{Introduction}

Supersoft X-ray sources (SSS) are a class of soft X-ray emitters whose spectra resemble a blackbody in the temperature range $20-100 \mathrm{eV}\left(3-7 \times 10^{5} \mathrm{~K}\right)$ with luminosities above $10^{35} \mathrm{erg} \mathrm{s}^{-1}$ (Greiner 1996). The first such sources were found with the Einstein Observatory by Long et al. (1981) and were later defined as a class after more were discovered with ROSAT (Truemper 1992; Kahabka et al. 1994; Greiner et al. 1991). The shape of the continuum is caused by atmospheric thermal emission, but blackbody models were initially used for spectral characterisations. The first SSS were found in the LMC where X-ray emission is subject to less photoelectric absorption along the line of sight than in the case of most Galactic SSS. In addition to the class of persistent SSS, classical novae $(\mathrm{CNe})$ have frequently been observed to emit an SSS spectrum during the later phase of their outburst. It is now commonly accepted that SSS emission originates in binary systems containing a white dwarf primary that hosts nuclear burning of material that is accreted from a secondary star; see van den Heuvel et al. (1992) and Kahabka \& van den Heuvel (1997), as well as Darnley et al. (2012) for discussion of types and the role of the secondary star in nova systems. In persistent SSS, the burning rate is roughly the same as the accretion rate (van den Heuvel et al. 1992; Kahabka \& van den Heuvel 1997), while in novae during outburst, the burning rate is higher, eventually leading the SSS emission to disappear once all hydrogen is consumed.

Before a nova explosion occurs, material from the companion is accreted and accumulates on the white dwarf surface. The pressure and temperature continuously increase until 
a thermonuclear runaway occurs, ejecting a high fraction of the accreted material, together with some core material. The ejecta initially form an expanding shell surrounding the white dwarf, which prevents high-energy radiation from escaping. $\mathrm{CNe}$ are optically bright until the density in the outer layers decreases, exposing hotter layers in the outflowing gases. The continuing shrinkage of the photosphere ultimately leads to a shift in the peak of the spectral energy distribution into the X-ray regime, producing an SSS spectrum when the photospheric radius is close to a white dwarf radius. SSS emission declines when nuclear burning ceases once all hydrogen in the burning zones is either consumed or ejected. For more details we refer to Starrfield et al. (2008).

Modelling SSS spectra is challenging, especially for novae. The SSS spectra observed in low resolution, e.g., with CCDs, can often be reproduced by a blackbody fit. While no meaningful physical parameters can be obtained from this approach, it has been shown that these systems are far from thermal equilibrium; see e.g. Balman et al. (1998). The physics contained in nonlocal thermodynamic equilibrium (NLTE) atmosphere models are more realistic, but CCD spectra are not adequate for constraining LTE nor NLTE parameters because the spectral resolution of CCDs does not match the complexity of atmosphere models (e.g. Hartmann \& Heise 1996, 1997). Parmar et al. (1998, 1997) have obtained different results from blackbody fits and NLTE atmosphere models, but the reproduction of the data is always the same. Page et al. (2010) and Osborne et al. (2011) have obtained similar effective temperatures from blackbody fits and NLTE atmosphere fits to Swift/XRT spectra. The true complexity of radiative transport models has often been ignored, with only the effective temperature, surface gravity, and some limited patterns in composition considered as variable parameters, but the higher resolution of X-ray grating spectrometers reveals that this approach is invalid (e.g. Sala et al. 2008, 2010).

With the availability of the grating spectrometers XMM-Newton RGS (Reflection Grating Spectrometer), and Chandra LETG/HETG (Low/High Energy Transmission Grating Spectrometers), simple fitting of atmosphere models with a small number of parameters is inadequate, and more sophisticated models are needed. Currently two approaches are being pursued. Hydrostatic NLTE models such as TMAP benefit from extensive experience and a mature understanding of NLTE approximations, opacities, and atomic physics (e.g. Rauch et al. 2010). However, considerable blue shifts of absorption lines in nova spectra as reported by, e.g., Ness (2010) are not reproduced by TMAP, requiring an artificial correction. Their importance is controversial because no clear signs of P Cyg profiles have been found in observed spectra, suggesting a low mass loss rate. A self-consistent approach is attempted with the wind model recently presented by van Rossum (2012) which is based on spherically symmetric, expanding, NLTE model atmospheres that have been applied by Hauschildt et al. (1992) to novae during their early stages. The possibility of line absorption caused by expanding clumps far from the photosphere needs to be tested and included in the atmosphere calculations if needed.

For this work, we bypass the complications of spectral models and concentrate on the data themselves in a comparative, qualitative study. Several X-ray grating spectra of SSS have been obtained, including the two prototypes Cal 83 and Cal 87 (Long et al. 1981; Crampton et al. 1987) and a number of $\mathrm{CNe}$ as well as recurrent novae $(\mathrm{RNe})$ that have been observed during their X-ray bright SSS phase. We extracted all available archival SSS $\mathrm{X}$-ray grating spectra to study commonalities between them and group them into two classes.
In Sect. 2 we describe the sample of targets and the observations that are discussed in this work. The data are presented in Sect. 3 and our interpretations plus implications of our results are presented in Sect. 4. We close with our conclusions and a summary in Sect. 5.

\section{Observations}

An overview of all targets discussed in this work is given in Table 1 . We only study SSS spectra, and exclude all novae that were not observed with an X-ray grating during their SSS phase. In addition other grating spectra were extracted for comparison. In Table 1, the sources are listed by class as defined in the footnotes, date of latest outburst for novae, system inclination angle if known, and distance. References for values taken from the literature are given in the footnotes. Measurements of inclination angle are not straightforward, yielding rather more secure measurements for higher inclination angles via observations of eclipses, illumination effects, or spectroscopic evidence. Distance measurements of Galactic sources beyond $\sim 1 \mathrm{kpc}$ are highly uncertain as no direct parallax measurements of the progenitor systems are available. The upcoming ESA mission Gaia will give more precise measurements but novae that have returned to their quiescent state may be too faint. Empirical methods such as maximum magnitude versus rate of decline (MMRD) relationships are frequently used but are often inaccurate. For more discussion and a collection of more system parameters, we refer to Schwarz et al. (2011).

The journal of X-ray observations used in this work is presented in Table 2 with columns: days after discovery for $\mathrm{CNe}$ and $\mathrm{RNe}$ relative to the respective reference times given in Table 1, observation identifier, grating instrument, start time of observation, and effective exposure time (after all corrections). The Chandra LETGS has been used in conjunction with the High Resolution Camera (HRC), except for the observation of Cal 87, where the Advanced CCD Imaging Spectrometer (ACIS) was used as the detector to record the dispersed photons. For novae, only those observations are included that were taken during the SSS phase. In the bottom of Table 2, T Pyx is also marked as an observation after the nova had turned off, along with V382 Vel and RS Oph. Further, we extracted an XMM-Newton spectrum of the obscured Active Galactic Nucleus (AGN) NGC 1068 (Kinkhabwala et al. 2002) for comparison.

Standard SAS 11.0 and CIAO 3.4 tools were used to obtain calibrated spectra from the raw XMM-Newton and Chandra data, respectively. Newer versions are available but re-extraction is only needed for quantitative analysis which is not pursued in this work. The count spectra are constructed by collecting the dispersed photons from optimised extraction regions. They are then converted to calibrated flux spectra by dividing the number of counts by the effective area in each spectral bin which is taken from the calibration of the respective instruments. For grating spectra, this procedure is justified because the response matrices are sufficiently diagonal to yield well-known photon energies for each spectral channel. While instrumental line broadening $(\sim 0.05 \AA)$ is not corrected, it is not important for the qualitative analyses performed in this paper.

Selected calibrated grating spectra are shown in Fig. 1. The absorbed blackbody curves are included in Fig. 5 only to guide the eye to the rough location of the continuum. Two RGS spectra of V723 Cas have been combined using the SAS task rgscombine, and all six Chandra spectra of RX J0513.9-6951 are co-added. 
Table 1. Description of targets.

\begin{tabular}{|c|c|c|c|c|}
\hline Target & Гype/sub-type ${ }^{a}$ & $t_{\mathrm{ref}}{ }^{b}$ & $i^{c}$ & $d^{d}$ \\
\hline \multicolumn{5}{|l|}{ Persistent SSS } \\
\hline Cal 83 & $\mathrm{SSS} / \mathrm{SSa}$ & - & $20-30^{1,2}$ & $48.1 \pm 2.9^{3}$ \\
\hline Cal 87 & SSS/SSe & - & $73-77^{4,5}$ & $48.1 \pm 2.9^{3}$ \\
\hline RX J0513.9-6951 & $\mathrm{SSS} / \mathrm{SS} a$ & - & $\sim 35^{6}$ & $48.1 \pm 2.9^{3}$ \\
\hline QR And ${ }^{e}$ & $\mathrm{SSS} / \mathrm{SSe}$ & $35-60$ & $7^{7} ;>79^{8,9,10}$ & $2.0^{10}$ \\
\hline \multicolumn{5}{|c|}{ Classical Novae (CNe) } \\
\hline $\mathrm{HV} \mathrm{Cet}^{f}$ & $\mathrm{CN} / \mathrm{SSe}$ & $2008-10-07.4$ & $\operatorname{high}^{11}$ ? & $4.5 \pm 2.0^{12}$ \\
\hline LMC 2012 & CN/SSa & 2012-03-26.4 & ? & $48.1 \pm 2.9^{3}$ \\
\hline V959 Mon & $\mathrm{CN} / \mathrm{SSe}$ & 2012-08-09.8 & $82 \pm 6^{16}$ & $1.5^{17}$ \\
\hline V1494 Aql & $\mathrm{CN} / \mathrm{SSe}$ & $1999-12-01.8$ & $78.5^{18,19,20}$ & $1.6 \pm 0.1^{21}$ \\
\hline V5116 Sgr & $\mathrm{CN} / \mathrm{SSa}, \mathrm{e}$ & 2005-07-04.0 & $\operatorname{high}^{24,26}$ & $11 \pm 3^{25}$ \\
\hline V723 Cas & $\mathrm{CN} / \mathrm{SSa}$ & $1995-08-24.0$ & $62^{26,27}$ & $3.9 \pm 0.2^{26}$ \\
\hline V382 Vel & $\mathrm{CN} / n e b$ & $1999-05-22.4$ & $25-67^{28}$ & $1.7 \pm 0.3^{29}$ \\
\hline V4743 Sgr & CN/SSa & 2002-09-19.8 & $?$ & $3.9 \pm 0.3^{15}$ \\
\hline $\mathrm{KTEri}^{g}$ & CN/SSa & 2009-11-14.6 & $58_{-7}^{+613}$ & $6.5^{14}$ \\
\hline V2491 $\mathrm{Cyg}^{g}$ & $\mathrm{CN} / \mathbf{S S a}$ & 2008-04-10.7 & $80_{-12}^{+322} ?$ & $10.5^{23}$ \\
\hline LMC $2009 \mathrm{a}^{g}$ & $\mathrm{RN} / \mathrm{SSe}$ & 2009-02-05.1 & $?$ & $48.1 \pm 2.9^{3}$ \\
\hline \multicolumn{5}{|c|}{ Recurrent Novae $(\mathbf{R N e})^{b}$} \\
\hline RS Oph & RN/SSa & $2006-02-12.8$ & $30-40^{30,31}$ & $1.6 \pm 0.3^{32,33}$ \\
\hline U Sco & RN/SSe & 2010-01-27.8 & $82^{34,35}$ & $12 \pm 2^{36}$ \\
\hline TPyx & $\mathrm{RN} / n e b$ & $2011-04-14.3$ & $<20^{37,38,39}$ & $4.8 \pm 0.5^{37,40}$ \\
\hline
\end{tabular}

Notes. ${ }^{(a)}$ CN: Classical Nova; RN: Recurrent Nova; SSS: persistent Super Soft Source. Sub-types are given indicating absorption-line dominated X-ray spectra (SSa: Sect. 3.2), emission line dominated spectra (SSe: Sect. 3.3), and post-outburst nebular spectra (neb: Sect. 3.6). The clearest cases are given in bold face while intermediate or less clear cases are given in italic. V5116 Sgr transitions between SSa and SSe, marked by SSa,e (Sect. 3.5). ${ }^{(b)}$ Discovery date for CNe and RNe used as reference time (latest outburst for RNe). ${ }^{(c)}$ Inclination angle in degrees. ${ }^{(d)}$ Distance (kpc); for LMC novae, the distance to the LMC is given. ${ }^{(e)}$ Aka RX J0019.8+2156. ${ }^{(f)}$ Aka CSS081007:030559+054715. ${ }^{(g)}$ Possible RN.

References. ${ }^{(1)}$ Crampton et al. (1987); (2) Schmidtke \& Cowley (2006); ${ }^{(3)}$ Macri et al. (2006); ${ }^{(4)}$ Schandl et al. (1997); (5) Ebisawa et al. (2001); ${ }^{(6)}$ Hutchings et al. (2002); (7) Becker et al. (1998) $(35<i<60)$; ${ }^{(8)}$ Kahabka (1996); ${ }^{(9)}$ Tomov et al. $(1998)(i>79)$; ${ }^{(10)}$ Beuermann et al. (1995); (11) Beardmore et al. (2012); (12) Schwarz et al. (2008); ${ }^{(13)}$ Ribeiro et al. (2013a); ${ }^{(14)}$ Ragan et al. (2009); (15) Vanlandingham et al. (2007); (16) Ribeiro et al. (2013b); (17) Munari et al. (2013); ${ }^{(18)}$ Bos et al. (2001); (19) Barsukova \& Goranskii (2003); (20) Hachisu et al. (2004); (21) Iijima \& Esenoglu (2003); ${ }^{(22)}$ Ribeiro et al. (2011); ${ }^{(23)}$ Helton et al. (2008); (24) Dobrotka et al. (2008); (25) Sala et al. (2008); (26) Lyke \& Campbell (2009); (27) Goranskij et al. (2007); ${ }^{(28)}$ Shore et al. (2003); ${ }^{(29)}$ Della Valle et al. (2002); ${ }^{(30)}$ Dobrzycka \& Kenyon (1994); (31) Ribeiro et al. (2009); ${ }^{(32)}$ Bode (1987); ${ }^{(33)}$ Barry et al. (2008); ${ }^{(34)}$ Schaefer (1990); ${ }^{(35)}$ Thoroughgood et al. (2001); ( ${ }^{36)}$ Schaefer (2010); ( ${ }^{37)}$ Chesneau et al. (2011); (38) Uthas et al. (2010); ${ }^{(39)}$ Sokoloski et al. (2013); ${ }^{(40)}$ Shore et al. (2011).

\section{Results}

Past X-ray observations of SSS have displayed a high degree of diversity of phenomena, and the objective of this work is to search for any systematic trends in the data. In light of the complexity of required models, which unavoidably comes with a high degree of uncertainty in model parameters, we base our approach directly on the data. In the following paragraphs, we show and describe SSS spectra of different systems.

\subsection{Commonalities between SSS spectra}

For comparison of SSS spectra from different systems or different evolutionary phases, we plot 2-3 spectra in the same graphs using a combination of lines and shadings of different colours as marked in the respective legends of Figs. 2-4, 7, and 9. Qualitative comparison of spectra is facilitated by rescaling if needed, compensating, e.g., for different intrinsic brightness or distance. Selected line transitions are labelled at their rest wavelengths without necessarily claiming the respective lines have been detected.

Ness et al. (2011) found a remarkable similarity between two SSS spectra of RS Oph and V2491 Cyg, taken on days 39.7 and 39.9 after their respective outbursts (see their Fig. 12). Both sources varied with time (Ness et al. 2007b, 2011), and we compared other SSS spectra of RS Oph and V2491 Cyg taken earlier and later during their respective evolution and found those to be different.

While Ness et al. (2011) reported fundamentally different spectra of RS Oph and V4743 Sgr, we show in Fig. 2 that one of five SSS spectra of V4743 Sgr is in fact remarkably similar to one out of four SSS spectra of KT Eri; note that no rescaling is needed! Both novae were observed multiple times, and their X-ray spectra varied on different time scales with V4743 Sgr evolving much slower. Their optical decline time scales show the same trend, yielding $t_{2}=9$ and 6.6 days for V4743 Sgr and KT Eri, respectively (Morgan et al. 2003; Hounsell et al. 2010). As in RS Oph and V2491 Cyg, both novae evolved differently, yielding a high degree of similarity only on days 180.4 and 84.6 of V4743 Sgr and KT Eri, respectively. The two pairs of novae RS Oph/V2491 Cyg and V4743 Sgr/KT Eri evolved differently but also passed through an identical spectral phase.

\subsection{The SSa subclass}

The SSS X-ray spectra of the four systems RS Oph, V2491 Cyg, V4743 Sgr, and KT Eri are dominated by a blackbody-like continuum with deep absorption lines (Fig. 13 in Ness et al. 2011 and Fig. 2 in this work). We chose these characteristics to define 
Table 2. Journal of X-ray grating observations.

\begin{tabular}{|c|c|c|c|c|c|c|c|c|c|}
\hline \multirow[t]{2}{*}{ Target } & \multirow[t]{2}{*}{ Day $^{a}$} & \multirow[t]{2}{*}{$\mathrm{ObsID}^{b}$ In } & \multirow[t]{2}{*}{ Instrument } & \multicolumn{2}{|c|}{ Exp.time } & \multirow{2}{*}{$\begin{array}{l}\mathrm{CR}^{c} \\
\mathrm{cts}^{-1}\end{array}$} & \multirow{2}{*}{\multicolumn{2}{|c|}{$\begin{array}{c}f_{T}{ }^{d} \quad f_{H}{ }^{e} \\
10^{-10} \mathrm{erg} \mathrm{cm}^{-}\end{array}$}} & \multirow{2}{*}{$\begin{array}{l}f_{S}{ }^{f} \\
\mathrm{~s}^{-1}\end{array}$} \\
\hline & & & & (UT) & $(\mathrm{ks})$ & & & & \\
\hline \multirow{5}{*}{\multicolumn{2}{|c|}{ Cal 83}} & 0077 & LETGS & $1999-11-29.27$ & 51.2 & & \multicolumn{3}{|c|}{ no detection } \\
\hline & & \multicolumn{2}{|c|}{0123510101 RGS } & $2000-04-23.84$ & 45.0 & 0.43 & 2.07 & 1.87 & 0.21 \\
\hline & & 1900 & LETGS & $2001-08-15.67$ & 35.2 & 0.09 & 2.18 & 1.81 & 0.37 \\
\hline & & 3402 & LETGS & 2001-10-03.48 & 61.4 & \multicolumn{4}{|c|}{ no detection } \\
\hline & & 050086060 & 01 RGS & $2007-11-24.90$ & 22.1 & 0.23 & 2.12 & 1.93 & 0.18 \\
\hline \multirow{2}{*}{\multicolumn{2}{|c|}{$\mathrm{Cal} 87$}} & \multicolumn{2}{|c|}{1896 ACIS/LETGS } & 2001-08-13.84 & 93.9 & 0.03 & 2.20 & 1.94 & 0.26 \\
\hline & & 015325010 & 01 RGS & 2003-04-18.87 & 77.6 & 0.12 & 0.61 & 0.32 & 0.28 \\
\hline \multirow{2}{*}{\multicolumn{2}{|c|}{ QR And }} & 0075 & LETGS & $2000-09-28.42$ & 51.4 & 0.01 & 0.99 & 0.65 & 0.35 \\
\hline & & 004794010 & 01 RGS & $2001-12-31.77$ & 56.6 & 0.07 & 0.42 & 0.34 & 0.09 \\
\hline \multicolumn{2}{|c|}{ RX J0513.9-6951 } & 3503 & LETGS & $2003-12-24.29$ & 47.7 & 0.08 & 1.75 & 1.46 & 0.29 \\
\hline & & 5440 & LETGS & $2005-04-20.80$ & 24.6 & 0.12 & 2.53 & 2.08 & 0.44 \\
\hline & & 5441 & LETGS & 2005-04-27.95 & 25.0 & 0.40 & 5.93 & 5.33 & 0.60 \\
\hline & & 5442 & LETGS & $2005-05-03.24$ & 25.5 & 0.39 & 5.84 & 5.26 & 0.57 \\
\hline & & 5443 & LETGS & $2005-05-13.82$ & 22.5 & 0.25 & 4.19 & 3.62 & 0.57 \\
\hline & & 5444 & LETGS & 2005-05-19.09 & 25.0 & 0.28 & 4.38 & 3.87 & 0.50 \\
\hline & & \multicolumn{2}{|c|}{ All combined ${ }^{h}$} & & 170 & 0.23 & 3.40 & 3.17 & 0.23 \\
\hline HV Cet & 72.2 & 9970 & LETGS & $2008-12-18.62$ & 34.8 & 0.22 & 3.35 & 2.74 & 0.61 \\
\hline KT Eri & 71.3 & 12097 & LETGS & 2010-01-23.91 & 14.9 & 11.52 & 164 & 163 & 0.76 \\
\hline & 79.3 & 12100 & LETGS & $2010-01-31.94$ & 27.9 & 77.73 & 1031 & 973 & 58.5 \\
\hline & 84.6 & 12101 & LETGS & $2010-02-06.27$ & 47.8 & 37.48 & 509 & 503 & 6.35 \\
\hline & 158.8 & 12203 & LETGS & $2010-04-21.45$ & 32.4 & 106.5 & 1564 & 1406 & 158 \\
\hline LMC 2009a & 90.4 & 061000030 & 01 RGS & $2009-05-06.43$ & 37.5 & 0.23 & 1.84 & 1.71 & 0.14 \\
\hline & 165.0 & 061000050 & 01 RGS & $2009-07-20.03$ & 56.7 & 1.80 & 16.8 & 14.4 & 2.41 \\
\hline & 196.5 & 060459030 & 01 RGS & 2009-08-20.59 & 14.0 & 2.10 & 16.2 & 13.4 & 2.84 \\
\hline & 230.0 & 060459040 & 01 RGS & 2009-09-23.02 & 51.0 & 1.23 & 11.0 & 9.42 & 1.56 \\
\hline LMC 2012 & 31.5 & 14426 & LETGS & 2012-04-26.91 & 20.0 & 2.12 & 25.7 & 15.4 & 10.3 \\
\hline V959 Mon & 116.0 & 15596 & LETGS & $2012-12-03.82$ & 24.9 & 0.15 & 2.58 & 1.88 & 0.70 \\
\hline V1494 Aql & 301.5 & 2308 & LETGS & $2000-09-28.29$ & 8.1 & 0.72 & 10.5 & 9.22 & 1.26 \\
\hline & 304.7 & 0072 & LETGS & $2000-10-01.42$ & 18.1 & 0.93 & 12.4 & 11.5 & 0.89 \\
\hline V2491 Cyg & 39.9 & 055227050 & 01 RGS & $2008-05-20.59$ & 39.2 & 12.6 & 90.1 & 67.2 & 22.9 \\
\hline & 49.6 & 055227060 & 01 RGS & $2008-05-30.35$ & 29.8 & 2.7 & 17.5 & 12.8 & 4.68 \\
\hline V4743 Sgr & 180.4 & 3775 & LETGS & $2003-03-19.40$ & 24.7 & 38.91 & 540 & 528 & 11.4 \\
\hline & 196.14 & 012772050 & 01 RGS & 2003-04-04.93 & 35.2 & 44.6 & 300 & 289 & 10.7 \\
\hline & 301.9 & 3776 & LETGS & $2003-07-18.90$ & 11.7 & 37.16 & 499 & 481 & 18.1 \\
\hline & 371.0 & 4435 & LETGS & 2003-09-25.99 & 12.0 & 19.78 & 276 & 266 & 9.68 \\
\hline & 526.1 & 5292 & LETGS & 2004-02-28.06 & 10.3 & 3.61 & 54.4 & 53.2 & 1.21 \\
\hline V5116 Sgr & 609.7 & 040560020 & 01 RGS & $2007-03-05.72$ & 12.8 & 2.1 & 17.5 & 16.4 & 1.09 \\
\hline & 781.8 & 7462 & LETGS & $2007-08-24.80$ & 34.8 & 0.27 & 4.09 & 3.57 & 0.53 \\
\hline V723 Cas & 5481.7 & 065207010 & 01 RGS & $2010-08-26.76$ & 50.1 & 0.06 & 0.40 & 0.33 & 0.07 \\
\hline & 6018.1 & 067349010 & 01 RGS & $2012-02-14.02$ & 90.0 & 0.07 & 0.35 & 0.27 & 0.08 \\
\hline & & All combin & ined $^{h}$ & & 140 & 0.07 & 0.32 & 0.26 & 0.07 \\
\hline RS Oph & 26.12 & 041018020 & 01 RGS & 2006-03-10.96 & 11.7 & 2.5 & 14.8 & 9.89 & 4.92 \\
\hline & 39.7 & 7296 & LETGS & $2006-03-24.52$ & 10.0 & 30.16 & 312 & 192 & 120 \\
\hline & 54.0 & 041018030 & 01 RGS & 2006-04-07.88 & 18.6 & 134 & 864 & 457 & 407 \\
\hline & 66.9 & 7297 & LETGS & $2006-04-20.73$ & 6.5 & 68.34 & 726 & 408 & 319 \\
\hline USco & 18.7 & 12102 & LETGS & 2010-02-14.49 & 23.0 & 0.38 & 5.57 & 4.89 & 0.68 \\
\hline & 22.9 & 065030020 & 01 RGS & $2010-02-19.65$ & 63.4 & 0.95 & 7.75 & 6.98 & 0.78 \\
\hline & 34.8 & 056158030 & 01 RGS & 2010-03-03.61 & 54.3 & 1.1 & 6.74 & 3.35 & 3.40 \\
\hline T Pyx & $203.6^{g}$ & 12401 & LETGS & 2011-11-03.86 & 39.8 & 0.09 & 1.92 & 1.31 & 0.61 \\
\hline V382 Vel & $267.9^{g}$ & 958 & LETGS & $2000-02-14.27$ & 24.4 & 0.13 & 2.08 & 1.34 & 0.74 \\
\hline RS Oph & $111.7^{g}$ & 7298 & LETGS & $2006-06-04.50$ & 20.0 & 0.13 & 2.29 & 1.25 & 1.04 \\
\hline NGC 1068 & & 011120010 & $\begin{array}{ll}01 & \text { RGS }\end{array}$ & $2000-07-29.73$ & 42.3 & For co & parisor & n Fig. & \\
\hline
\end{tabular}

Notes. ${ }^{(a)}$ After $t_{\text {ref }}($ Table 1$) ;{ }^{(b)}$ observation Identifiers; those shown in bold indicate observations used for Fig. 1 ; ${ }^{(c)}$ count rate per second; ${ }^{(d)}$ flux over total band 7-38 $\AA^{(e)}$ flux over hard range 7-23 $\AA ;{ }^{(f)}$ flux over soft range $23-38 \AA$; ${ }^{(g)}$ X-ray observation taken after nova had turned off. (h) for RX J0513.9-6951 and V723 Cas, all available observations were combined. 
J.-U. Ness et al.: Obscuration effects in SSS

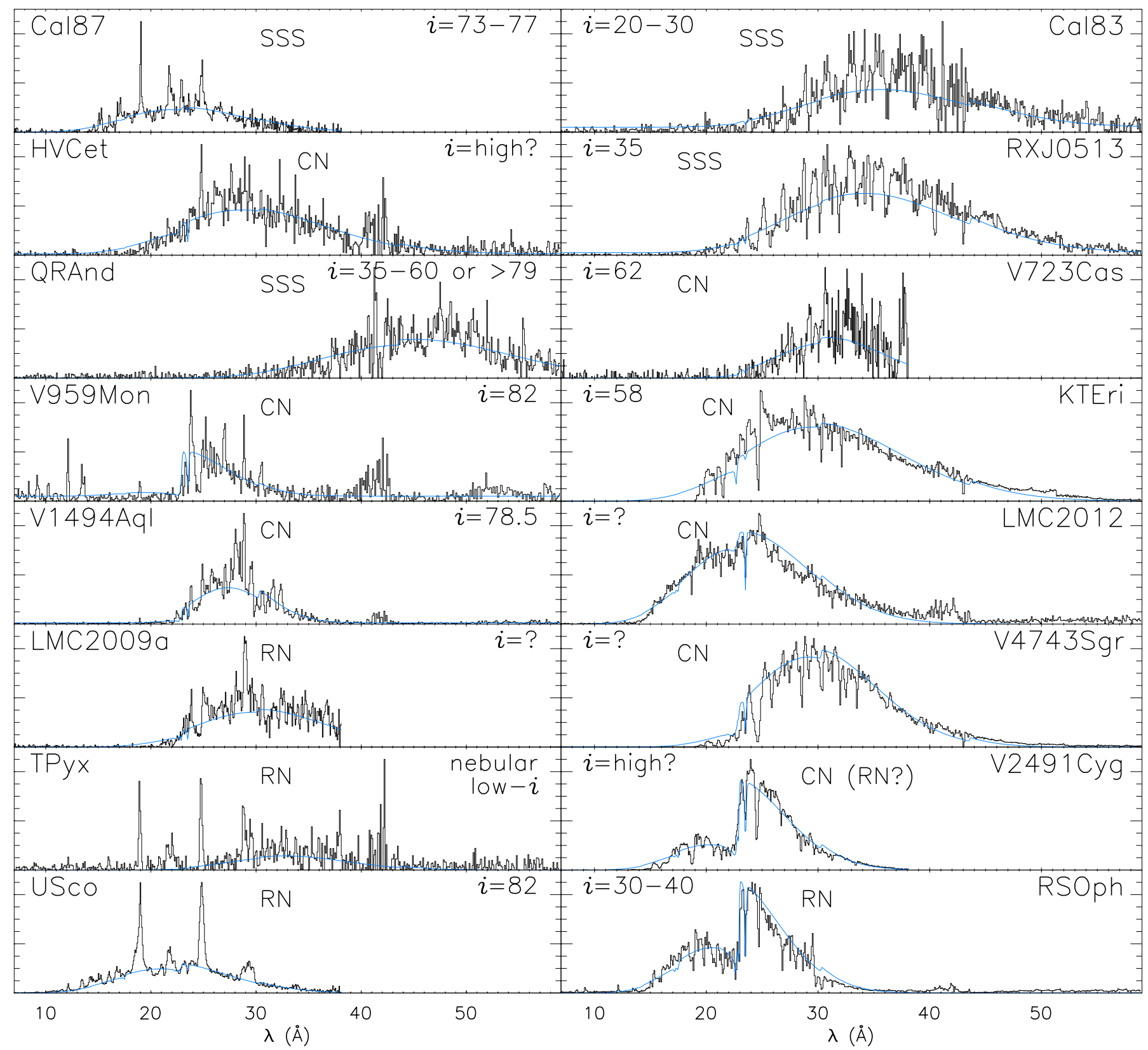

Fig. 1. High-resolution X-ray spectra in arbitrary flux units of SSS that contain emission lines without absorption features (SSe, left column) and those that consist of continuum and absorption lines (SSa, right column). Inclination angles $i$ from the literature (if known) are given (see Table 1). The blue thin lines are absorbed blackbody curves, indicating the presence of photospheric emission in all cases. The labels SSS, CN, and RN denote persistent supersoft sources, Classical Novae, and Recurrent Novae, respectively. An apparent emission feature around $40 \AA$ in some Chandra spectra is an instrumental artefact owing to division by low effective areas. All six Chandra spectra of RX J0513.9-6951 (second panel in right column) are co-added, and for V723 Cas (third panel in right column), the two XMM-Newton spectra have been combined with rgscombine.

a new subclass, SSa (a for absorption lines). After inspection of a Chandra grating spectrum of the recent nova LMC 2012, we also group this nova into this subclass. In the left column of Fig. 1, eight examples of SSa spectra are shown.

Another object that could be grouped into the SSa subclass is Cal 83 but the spectrum is not as well exposed (owing to the large distance to the LMC). An NLTE atmosphere model by Lanz et al. (2005) could be fit to the Chandra spectrum. Also the four SSa spectra of V4743 Sgr, V2491 Cyg, RS Oph, and KT Eri have been used to adjust preliminary atmosphere models with some success (Rauch et al. 2010; van Rossum 2012; Ness et al. 2011; Nelson et al. 2008) while for some other observations, spectral modelling has so far not been successful, e.g.
V1494 Aql (Rohrbach et al. 2009). Possibly, all SSa spectra can be reproduced by atmosphere models, in which case Cal 83 is also an SSa, while V1494 Aql may not be an SSa.

With few exceptions, emission lines cannot easily be discerned in the SSa subclass. The emission lines seen in RS Oph on day 39.7 (Ness et al. 2007b) need to be interpreted in the context of the cooling shock that occurred between the stellar wind of the companion and the nova ejecta. Schönrich \& Ness (2008) report that those lines above the SSS continuum appear enhanced compared to those at shorter wavelengths, indicating line pumping. For nova LMC 2012, Takei et al. (2012) report detections of emission lines, and also the SSa spectrum of KT Eri contains additional emission lines (Fig. 2). We consider a strong 

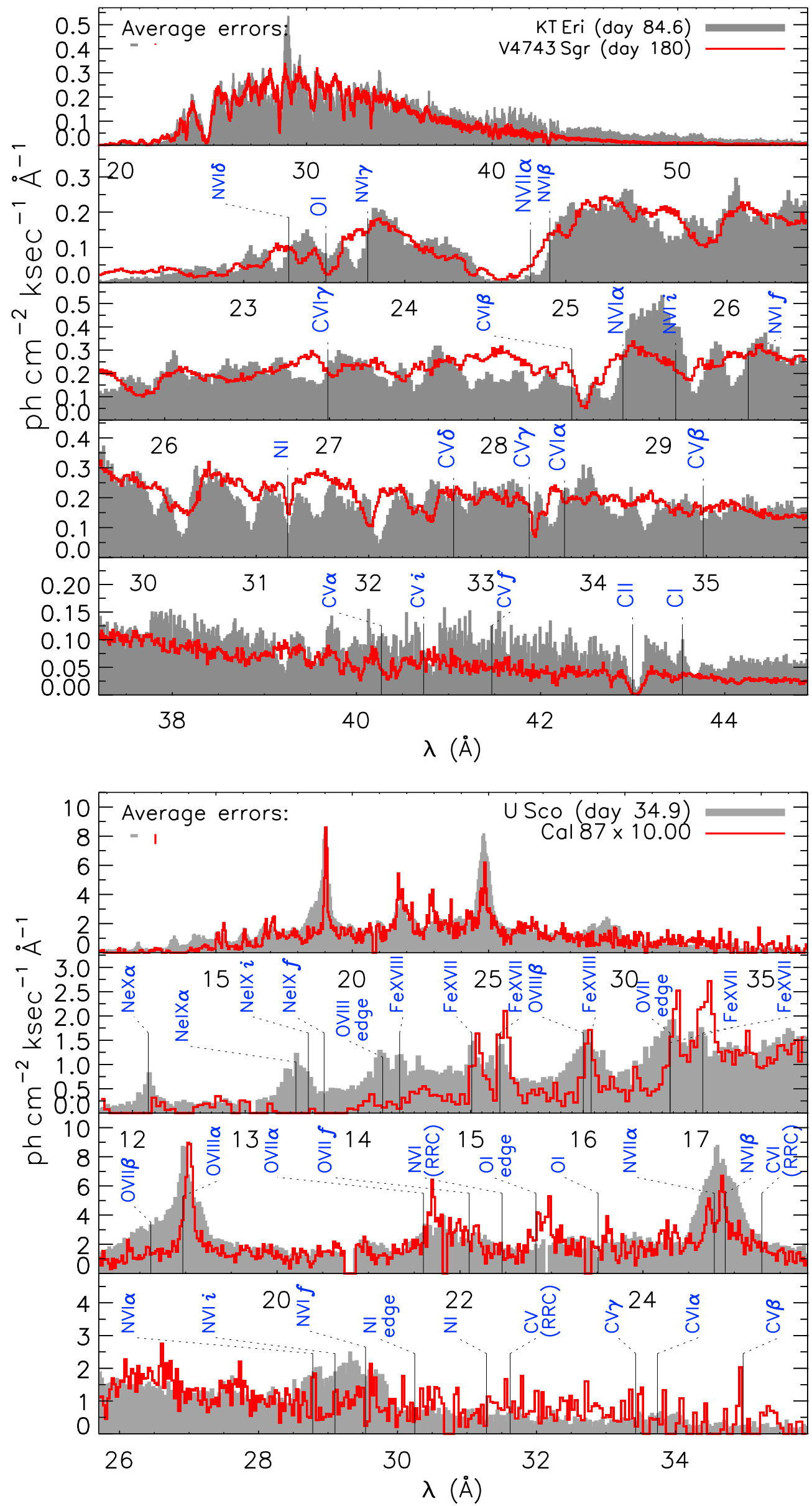

Fig. 2. Comparison plot of grating spectra. Examples of the SSa class: comparison of high-resolution X-ray spectra of the two CNe KTEri and V4743 Sgr. No scaling is necessary to fit both spectra in the same graph with the same units. In this figure as well as in Figs. 3, 4, 7, and 9, the top panel shows the entire wavelength range and legends with average error bars for each observation (left) and line/fill styles belonging to the corresponding target names (right) where the days in brackets are days after $t_{0}$ in Table 2. The panels below show details, with some overlap spectral ranges. Prominent boundbound transitions are marked with vertical lines at their rest wavelengths, and a label on top of each line gives the corresponding element/ion descriptor.
Fig. 3. Examples of the SSe class: comparison of high-resolution X-ray spectra of U Sco and of the prototype SSS Cal 87 , scaled by a factor 10 in brightness. (The concept is the same as described in Fig. 2.) 


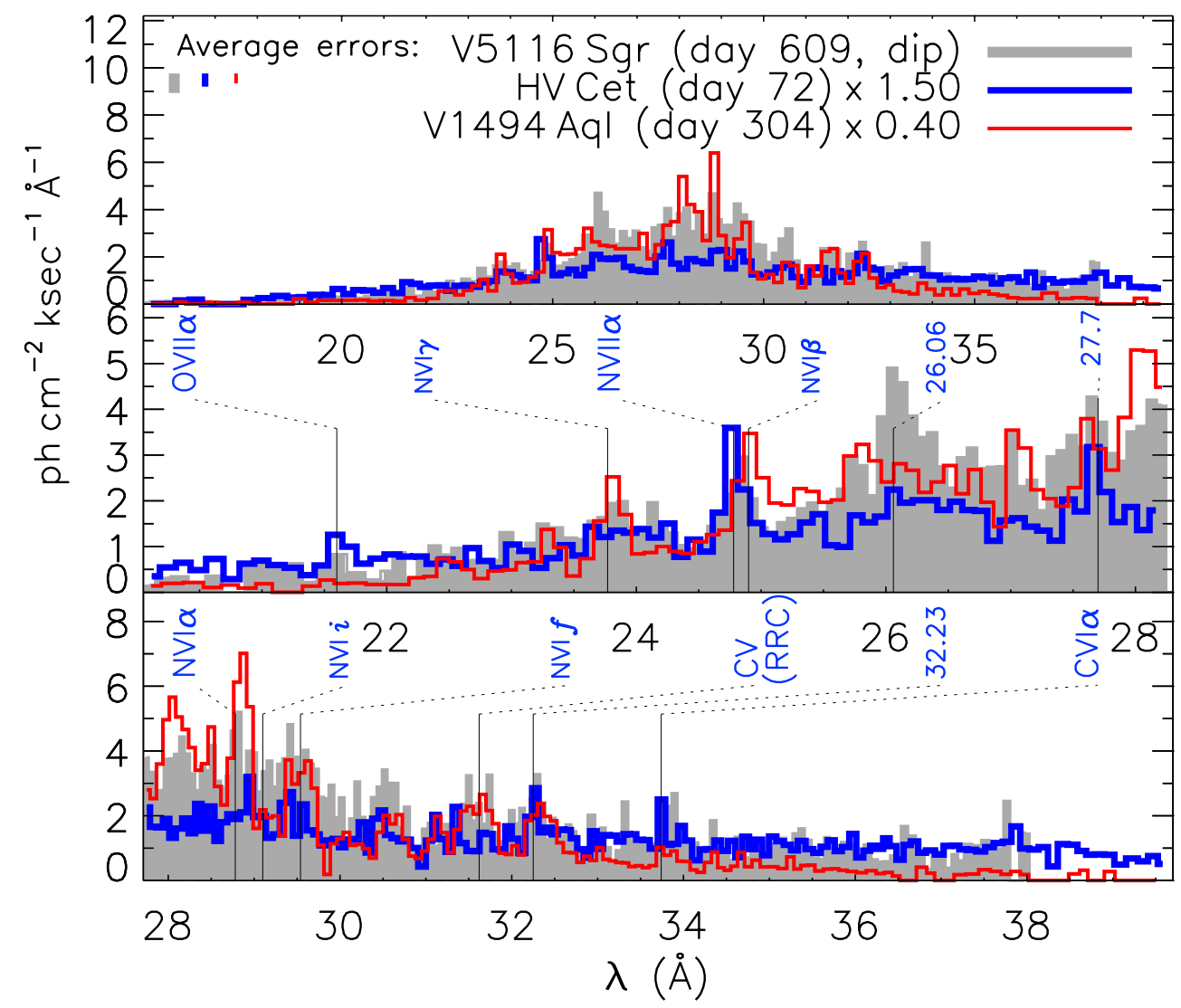

Fig. 4. Examples of intermediate cases: comparison of high-resolution X-ray spectra of the CNe V5116 Sgr, HV Cet, and V1494 Aql. The wavelengths of important transitions are marked and labelled. Lines found in other nova spectra in absorption but without having been identified are included with their wavelength values. (The concept is the same as described in Fig. 2.) possibility that some emission lines are always present in SSS spectra, albeit not easy to see in SSa spectra.

The presence of emission lines complicates spectral modelling with atmosphere models. More refined dynamic atmosphere models including mass loss will reveal whether the emission lines are part of P Cygni profiles (which cannot clearly be decided visually), or whether they originate from outside of the atmosphere, requiring an independent model component such as photoionised plasma.

In summary, the definition of a member of the SSa class is an $\mathrm{X}$-ray spectrum that contains a blackbody-like continuum with clearly visible absorption lines. Additional emission lines may be present but difficult to see within the complex atmospheric SSa spectrum.

\subsection{The SSe subclass}

During a detailed analysis of XMM-Newton observations of U Sco, Ness et al. (2012a) noted that this edge-on system resembles the SSS Cal 87. This is demonstrated in Fig. 3 where the XMM-Newton grating spectrum of U Sco taken on day 34.9 is shown in shades of grey and Cal 87 , scaled by a factor of 10 , as a red histogram line. The strongest lines seen in U Sco are also seen in Cal 87 but differ in their width. The Ne lines at $12 \AA$ and $13.5 \AA$ are only seen in U Sco while several Fe lines are present in both sources.

The strengths of the emission lines seem to be related to the strength of the underlying continuum at the wavelengths where they arise. This can be seen in Fig. 3 and also in Fig. 4 in Ness et al. (2012a).

The spectra of these two objects are the clearest examples of those that differ from the subclass of SSa, and therefore, we introduce the new subclass SSe, which are SSS spectra that contain strong emission lines above a weak continuum. The spectra of Cal 87 and U Sco shown in Fig. 3 contain no clear signs of absorption lines. Ness et al. (2012a) concluded from the presence of blackbody-like continuum emission during times of eclipse in U Sco that it results from Thomson scattering in the electronrich, ionised nova ejecta.

In summary, the definition of a member of the SSe class is an X-ray spectrum that contains a weak blackbody-like continuum without absorption lines and exhibits emission lines that are at least comparable in strength to the continuum. The emission lines in SSe are strongest where the continuum is strongest.

\subsection{Intermediate cases}

The spectral analysis of the grating spectra of the CN V1494 Aql presented by Rohrbach et al. (2009) turned out to be extremely difficult, and no satisfactory model was so far found. Some of the nova X-ray spectra contain a complex mixture of emissionand absorption lines which is illustrated with three examples in Fig. 4. Emission lines can be seen to occur in all three spectra, including some unidentified lines that arise at the same wavelengths were absorption lines were seen in the SSa spectra of V2491 Cyg, RS Oph, and V4743 Sgr (Ness et al. 2011). The three spectra in Fig. 4 are sufficiently similar to conclude that these sources belong to the same subclass, and their characteristics place them more into the SSe subclass, although these cases are not as clear as U Sco and Cal 87 (Fig. 3). Eight examples of SSe are shown in the left column of Fig. 1.

\subsection{Transitions between SSa and SSe}

The CN V5116 Sgr was highly variable during the XMM-Newton observation taken on day 609.7 (Sala et al. 2008, 2010). During 
an interval of $\sim 2 \mathrm{~h}(2 / 3$ of the orbit), the integrated brightness was more than an order of magnitude fainter than at the beginning of the observation, and during this time, emission lines were present as reported by Sala et al. (2010). This could be an example of an SSS switching between SSa and SSe states, possibly due to a temporary obscuration event (Sala et al. 2008).

We have studied four novae with variable X-ray light curves to investigate whether they have transitioned between SSa and SSe and illustrate the outcome in Fig. 5. From the light curves, we extracted spectra from time intervals of brighter emission (shown in the left) and fainter time intervals (right). To guide the eye, blackbody curves are included with blue thin lines.

The most extreme case is V4743 Sgr (top) in which all continuum emission was completely obliterated, leaving only emission lines (Ness et al. 2003). We speculate that the source of continuum X-rays either temporarily turned off or was completely obscured leaving us with emission from only the ejected gas.

V2491 Cyg was observed twice by XMM-Newton with a 3-h dip in the first observation (see Fig. 10 in Ness 2012). Ness et al. (2011) subdivided this observation into three time intervals a, b, c, and in the second row of Fig. 5, we show parts c (panel $3 L$ ) and $\mathrm{b}(3 R)$. The grey shaded spectrum in panel $3 R$ was taken 10 days later. The dip spectrum and the later one are about equally bright but differ decisively between $23-30 \AA$, with the dip spectrum having some characteristics of an SSe spectrum while the later spectrum is an SSa spectrum. The excess in the dip spectrum could have arisen further outside while the central continuum source was temporarily obscured.

The Chandra grating observation of RS Oph taken on day 39 after outburst was variable (see Fig. 7 in Ness 2012). This observation was taken during an episode of high-amplitude variations discovered by Osborne et al. (2011) in the Swift light curve. The high- and low-state spectra of RS Oph shown in the bottom of Fig. 5 are the same as those shown in Fig. 9 in Ness et al. (2007b). Excess emission is not as obvious, and this may not be a transition between SSa and SSe. The similarity of the brightness and hardness light curves noted by Ness et al. (2007b) in their Fig. 8 suggests that internal processes (as opposed to external obscuration) may lead to the variability, perhaps similar to the on- and off states in RX J0513.9-6951 (Schaeidt et al. 1993; Burwitz et al. 2008; Southwell et al. 1996; McGowan et al. 2005) or in Cal 83 (Kahabka 1997; Alcock et al. 1997; Rajoelimanana et al. 2013).

\subsubsection{Comparison of SSa and SSe spectra}

SSS spectra observed in grating resolution contain a multitude of complex features that no coherent model can currently reproduce. In order to put the dominant emission lines in SSe spectra into context with the more complex SSa spectra, we show in Fig. 6 direct comparisons between SSa and SSe spectra. The continuum component of an SSe spectrum is first estimated via a blackbody fit and then subtracted from the same observed SSe spectrum. The remaining emission line component is then added to the continuum component of an SSa spectrum that was also determined via blackbody estimation. We performed this operation for the bright/faint spectra of V5116 Sgr (top of Fig. 6) and for the SSa/SSe V2491 Cyg and U Sco (bottom of Fig. 6).

For both cases, one can see that the emission lines in SSe spectra are comparable in strength to various features in $\mathrm{SSa}$ spectra. Emission lines appearing so dominant in SSe may thus be present in SSa spectra as well but are hidden between
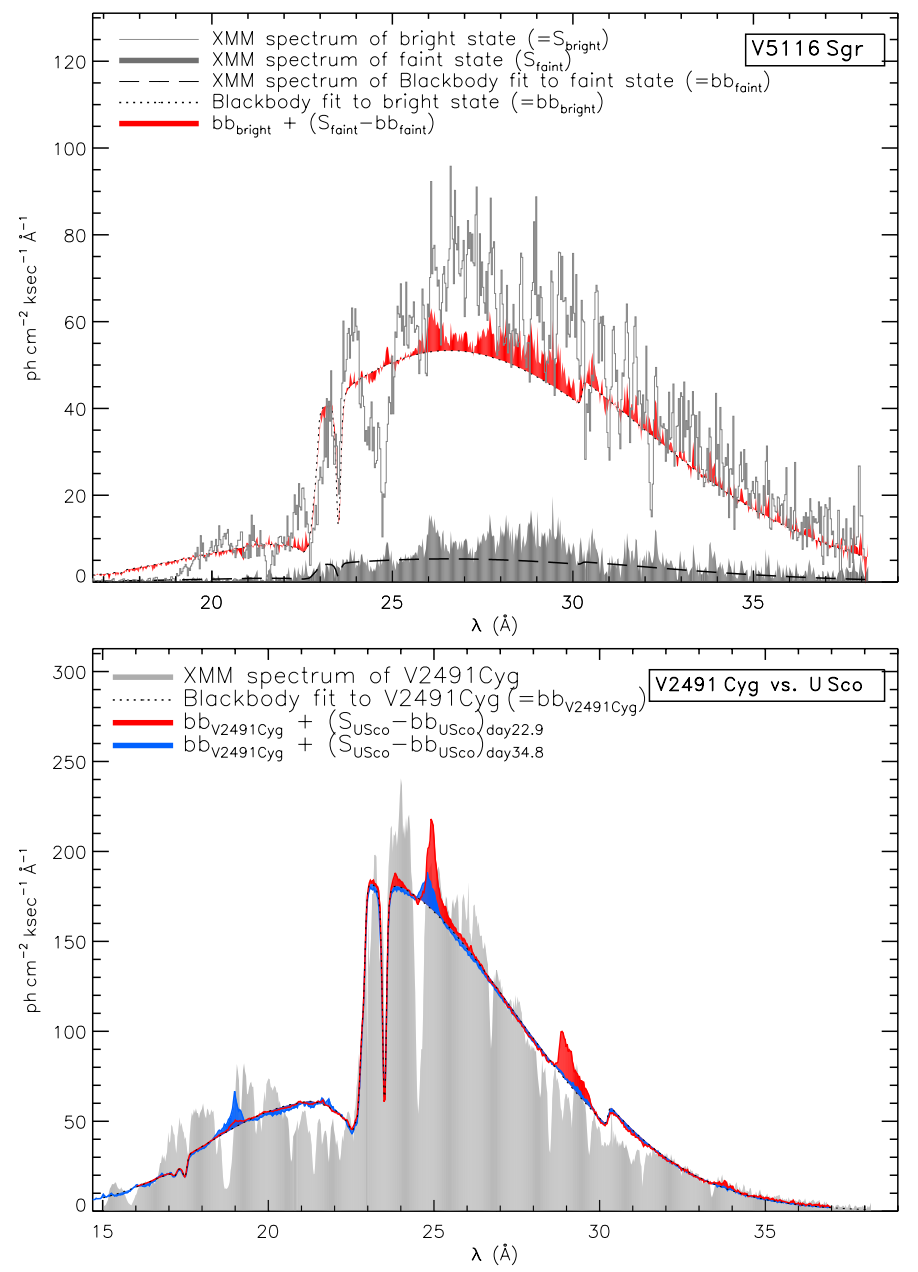

Fig. 6. Comparison of SSe and SSa spectra for the high/low state spectra of V5116 Sgr (top) and the two best examples of SSa, V2491 Cyg, and $\mathrm{SSe}, \mathrm{U}$ Sco, (bottom). The emission line component in SSe spectra, obtained by removing the weak continuum component, is added the continuum component of an SSa spectrum. The continua are approximated by blackbody models. The SSe emission line components are comparable in strength to complex atmospheric absorption/emission features in SSa.

complex atmospheric features. Without the complex continuum, the emission line component can be seen much better.

\subsection{Pre-SSS and post-outburst emission lines}

Emission lines can arise in different contexts. Before the start of the SSS phase in several novae, emission line spectra had frequently been observed with CCD spectrometers. While their spectral resolution is not high enough to resolve emission lines, the nature as emission line spectra has been established by means of collisional equilibrium models that reproduce these spectra (e.g., Krautter et al. 1996; Balman et al. 1998; Mukai \& Ishida 2001; Orio et al. 2001; Ness et al. 2009a). Distinct differences to SSe spectra are:

(1) The continuum component in collisional equilibrium originates from bremsstrahlung, which has a different shape from a blackbody.

(2) The emission line fluxes increase in strength during the SSS phase (Schönrich \& Ness 2008). For the SSe U Sco, only 


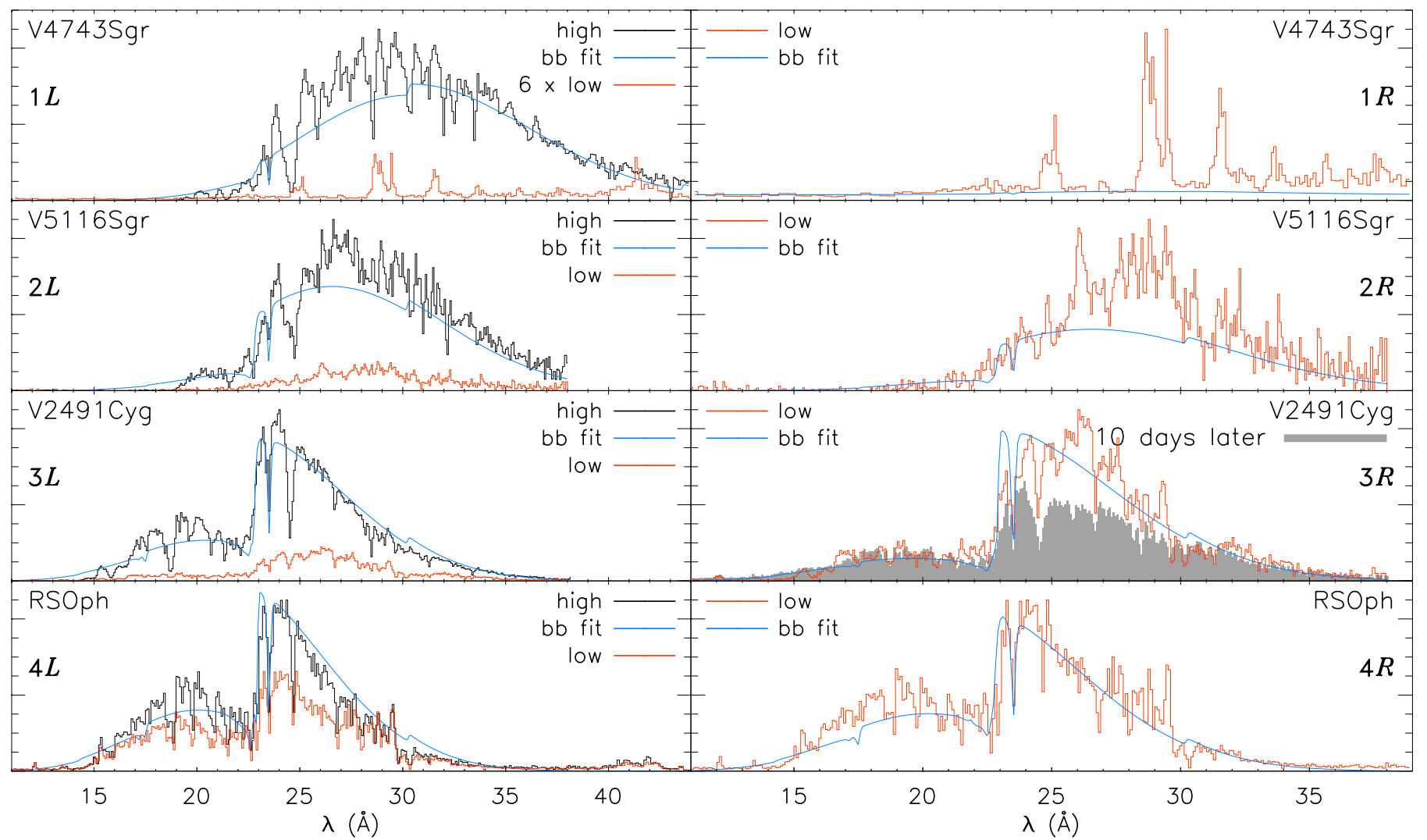

Fig. 5. Transitions between SSa (left) and SSe (right) states. High-resolution X-ray spectra, in arbitrary flux units, for four novae with variable light curves. The left column contains SSa spectra extracted from bright time intervals while the right column contains SSe spectra resulting from fainter time intervals. The light blue lines are absorbed blackbody curves that guide the eye to the rough location of the continuum. Only RS Oph (bottom) does not transition from SSa to SSe during the faint phase. Panel $3 R$ shows in grey shades the second XMM-Newton RGS observation of V2491 Cyg taken 10 days later, in comparison with the low-state spectrum. The low-state spectrum is more of SSe type while the later spectrum is more of SSa type. We believe that during times of fainter emission, less continuum is seen, leaving more emission originating further outside.

extremely faint early hard emission was seen (Schlegel et al. 2010).

(3) The emission lines in pre-SSS spectra are in most cases too faint for detection in grating spectra; exceptions are V959 Mon (Ness et al. 2012b) and symbiotic novae such as RS Oph (Ness et al. 2009b).

(4) The early hard X-ray component fades with time (Ness et al. 2009a), leaving even less potential contributions to SSe spectra.

The spectral changes with the start of the SSS phase have serendipitously been observed in an XMM-Newton observation of RS Oph on day 26.12 after the 2006 outburst (see Table 2) in which Nelson et al. (2008) discovered the appearance of a new soft component and showed that this new component is not yet atmospheric emission from the white dwarf but a complex emission line spectrum. The time evolution is illustrated in Fig. 8. About one hour after the start of the observation, an increase in count rate had occurred that Nelson et al. (2008) identified as a new soft component consisting of a complex emission line spectrum. The transitions belonging to the emission lines between $\sim 25-29 \AA$ are currently unknown, and our spectral time map shows that they were transient in nature, lasting less than one hour. At the same time as they disappeared, the N VII 1s2p (Ly $\alpha, 24.78 \AA$ ) line increased by a substantial amount, apparently the same amount of flux contained in the transient emission lines, preserving the integrated count rate (see right panel). The C VI Ly $\alpha$ line is not present, suggesting that none of the transient emission lines originates from carbon, even if line shifts are assumed as proposed by Nelson et al. (2008). It is possible that they all arise from nitrogen, although no transitions of nitrogen are known in this wavelength range. While a more coherent interpretation of these transient emission lines is beyond the scope of this paper, the relevant conclusion for this work is that the pre-SSS emission lines at soft wavelengths have become significantly stronger with the start of the SSS phase while harder emission lines have remained the same; see Fig. 6 in Ness (2012). These could be SSe features that are later hidden between complex atmospheric structures in SSa spectra.

For the four reasons above, we do not consider the emission lines in SSe as continuation of the early emission lines, and so, in particular for the persistent SSS Cal 87, a different mechanism is needed. We have restricted the definition of SSe in Sect. 3.3 to the presence of emission lines that coincide in strength with an underlying blackbody-like continuum.

Post-outburst X-ray emission line spectra arise in the radiatively cooling ejecta which has been shown for the post-outburst grating spectra of V382 Vel by Ness et al. (2005) and of RS Oph by Ness et al. (2009b). These two grating spectra are shown in Fig. 7 in comparison to a Chandra spectrum of the RN T Pyx, taken 203.6 days after the 2011 outburst (Tofflemire et al. 2011). The three spectra are qualitatively similar, suggesting a similar origin. Since the Chandra spectrum of TPyx was taken during the decline of the SSS phase, this spectrum may be a post-outburst spectrum that could arise from radiatively cooling ejecta. 
While pre- and post-SSS X-ray spectra are very similar in their appearance, their origin may still be different. The presence of high-velocity, dense ejecta during the early evolution of a nova facilitates shocks with the environment or, in the case of internal inhomogeneities, shocks within the ejecta. After the SSS phase has ended, the ejecta have dispersed, leaving only radiative cooling of optically thin plasma as X-ray production mechanism. Both are collisional processes, allowing the same spectral models to be applied, giving only different parameters (such as lower electron temperature in post-SSS spectra).

We do not group post-outburst emission line spectra into the SSe class because the emission line strengths do not scale with the strength of the respective underlying SSS continuum. In V382 Vel, the SSS spectrum during the active phase did not exceed $0.6 \mathrm{keV}$ (20 ̊) (Orio et al. 2002; Burwitz et al. 2002b,a) while in Fig. 7, emission lines are also seen at shorter wavelengths. The situation is similar for RS Oph, and in both systems, an early hard component had been present before the start of the SSS phase (Mukai \& Ishida 2001; Ness et al. 2009b).

\subsection{Dependence on inclination angle}

The arrangement of the spectra in Fig. 1 is chosen to display SSe in the left and SSa spectra in the right, although not all cases can unambiguously be classified (see Sect. 3.4). We include the inclination angles (if known) from Table 1 in the corners opposite to where the source names are given. As discussed above, higher inclination angles are more securely determined, yielding more high values than low values. All inclination angles above $\sim 70^{\circ}$ can be found in the left column, indicating that SSe spectra are a common phenomenon in high-inclination systems. The only exception may be the SSa V2491 Cyg that was proposed by Ribeiro et al. (2011) to be a high-inclination system, however, this has so far not been confirmed by observations of eclipses.

The CN V723 Cas is viewed at an intermediate inclination angle and contains a mixture of SSe and SSa emission, suggesting that there is a trend with inclination angle. The spectrum resembles that of QR And which lies at a similar inclination angle.

\section{Discussion}

Based on the observed sample of SSS spectra, we introduced the two sub-types SSa and SSe with the primary observational characteristics being the predominance of absorption lines versus emission lines, respectively (see definition at end of Sects. 3.2 and 3.3). Some sources transition between SSa and SSe states, yielding SSe spectra during episodes of fainter emission. We have shown an influence of inclination angle on the observed type of SSS spectrum. All systems with known high inclination angle (which is more securely measurable) display SSe spectra, while for many SSa spectra, the inclination angle is not known or not robustly confirmed. Emission lines in SSe spectra appear comparable in strength to complex sub-structures in SSa spectra, suggesting that they are always present and can better be seen when the atmospheric continuum with its complex structure is not present. SSe spectra may be the result of obscuration of the continuum component, exposing emission lines that probably originate from further away from the white dwarf.

Ebisawa et al. (2001) argued that at the given high inclination angle in Cal 87, the central source was blocked by the accretion disc, and scattering through the accretion disc corona could explain the observed spectrum (see also Ebisawa et al. 2010). Similarly, Beardmore et al. (2012) argue that the apparently sub-Eddington white dwarf luminosity in HV Cet is due to permanent obscuration of X-rays by the accretion disc rim. A smooth periodic X-ray modulation of nova V959 Mon (Page et al. 2013) also makes it likely that this source is viewed at high inclination and has a white dwarf surrounded by a scattering region.

In the following we discuss the origin of the continuum and the emission line components (4.1), obscuration effects in SSe (4.2) and resulting implications to determination of luminosities (4.2.2). Since most SSe spectra originate from high-inclination systems, the accretion disc likely plays a vital role but the novae in our sample do not necessarily possess an accretion disc. We discuss in Sect. 4.3 whether the disc has been destroyed during the initial nova explosion and how it could reform. Our main conclusion is that SSe are seen when a significant fraction of the photospheric emission is obscured, but not all X-ray variability is necessarily due to obscurations (see Sect. 4.4).

\subsection{Origin of the continuum and emission lines in SSe}

The continuum component in all SSS spectra is produced by the photosphere of the white dwarf. A photospheric temperature of several $10^{5} \mathrm{~K}$ implies $R \approx 10^{-2} R_{\odot}$. The U Sco system configuration requires the entire white dwarf be completely eclipsed, but Ness et al. (2012a) found the continuum emission during eclipse centre reduced by only $50 \%$. The only way to see this continuum emission also outside of the binary orbit is Thomson scattering that preserves the spectral shape. Thomson scattering can likely also explain the presence of some if not all continuum emission in SSe.

The origin of the emission lines on top of a blackbody-like continuum in SSe spectra is less clear. As discussed in Sect. 3.6, we do not consider the emission lines in SSe as a continuation of early emission lines sometimes seen in novae.

The strongest emission lines in SSe arise at wavelengths where the continuum is strongest. This is not typical of collisional equilibrium spectra and is rather suggestive of photoexcitation which should not be confused with photoionisation that has been disfavoured by Orio et al. (2013) for U Sco. Ions in excited states re-emit previously absorbed photons in all directions, independently of the location of the ionising source. Continuum photons emitted from the white dwarf into polar directions can effectively be scattered into other directions via this process. Because it is most efficient for resonance lines with high oscillator strengths, it is called resonant line scattering and has been proposed for Cal 87 by Greiner et al. (2004) and for U Sco by Ness et al. (2012a). Depending on the geometry of the scattering medium, photons can be scattered into the line of sight or out of the line of sight. Since these processes balance out in a spherically symmetric scattering medium, it needs to be asymmetric to allow for observable effects of resonant line scattering.

The contribution of resonant scattering to the emission lines dominates that of recombination when the column density through the absorber/re-emitter is low, but decreases as the column increases (Kinkhabwala et al. 2002). The detection of Radiative Recombination Continuum $\left(\mathrm{RRC}^{1}\right)$ features is a sign of recombination, but note that RRCs can be detected only for low temperatures. If there is additional heating (on top of photoheating), which brings the gas to higher temperatures (e.g., exceeding $\sim 30 \mathrm{eV}$, and dependent on spectrometer used and $\mathrm{S} / \mathrm{N}$ ),

1 RRCs are emission features reflecting the released energy during recombination. 


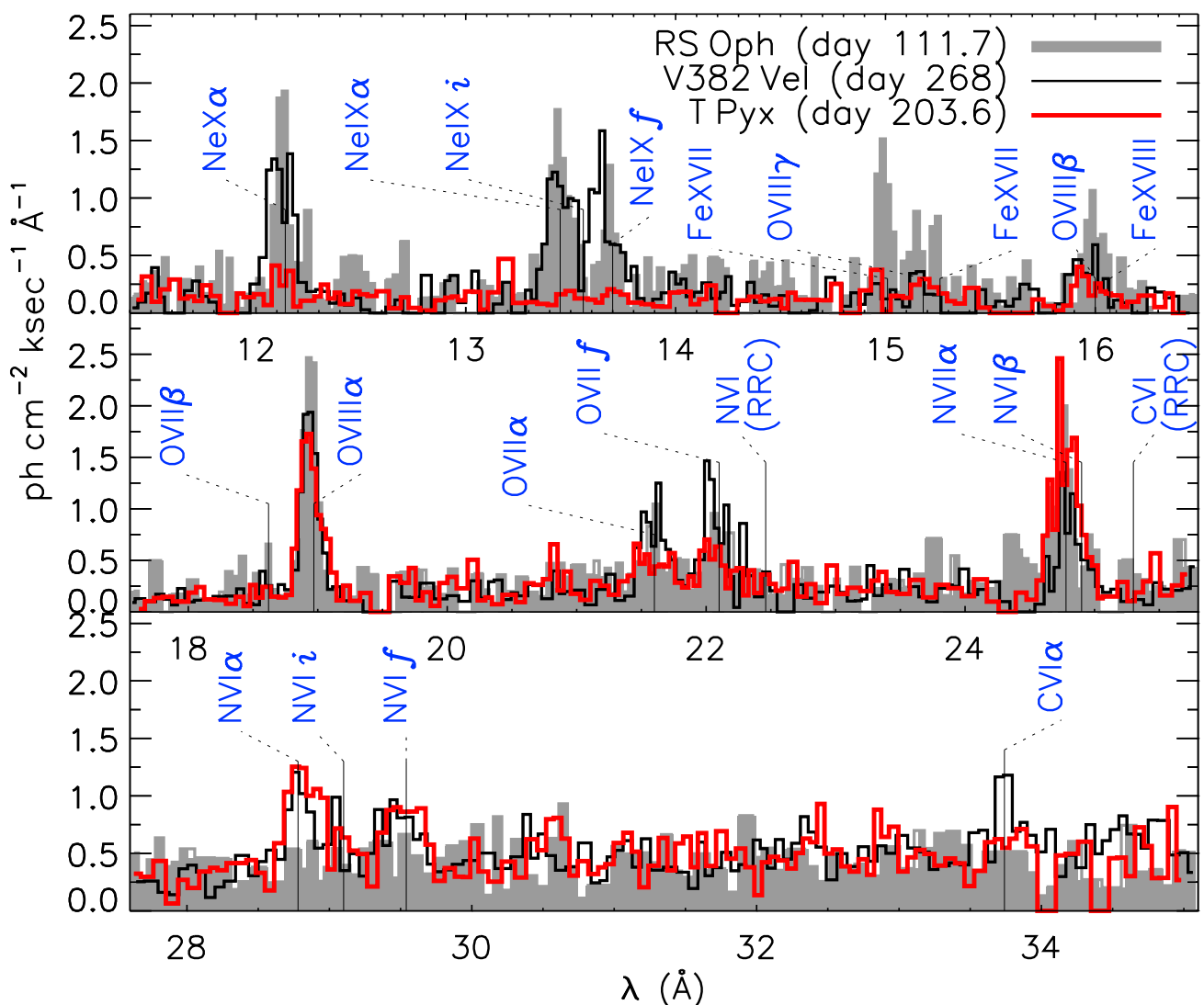

Fig. 7. Comparison of the postoutburst high-resolution X-ray spectra of V382 Vel (think black histogram), RS Oph (that was an SSa during outburst, thick red histogram), and the low-inclination system T Pyx (grey shadings). The emission lines in V382 Vel and RS Oph originate in the radiatively cooling ejecta after the novae had turned off. (The concept is the same as described in Fig. 2.)

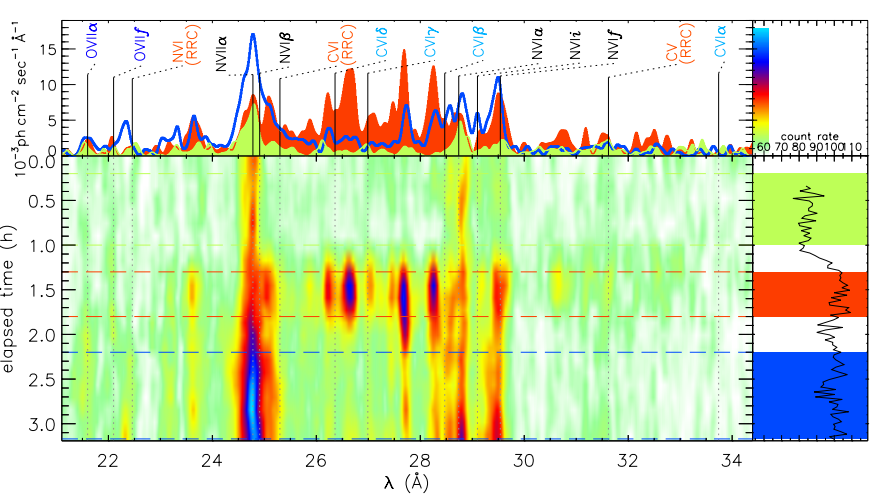

Fig. 8. Transition into the SSS phase observed with XMM-Newton in RS Oph starting day 26.12 after the 2006 outburst. The EPIC/pn light curve is shown to the right with three coloured shades marking time intervals from which the spectra in the top have been extracted. Line labels that belong to strong transitions in nitrogen (black), carbon (light blue), and oxygen (dark blue) are included, as well as Radiative Recombination Continuum (RRC) features in red. The central image is a brightness spectral/time map that uses a colour code from light green to light blue representing increasing flux values as shown with the colour bar along the spectral flux axis in the top right. See also Fig. 6 in Ness (2012).

the RRCs would be broadened in the spectrum beyond recognition. In that case, the plasma would be more collisional.

If the lines are the result of a mixture of processes, then it is difficult to explore them for quantitative diagnostics. In the case of pure resonance scattering, the line intensity is a measure of how the scattering medium covers the source. Based on the atomic cross section for the resonant Ly $\alpha^{2}$ transitions, for significant line absorption and scattering, an ionic column density

\footnotetext{
2 1s-2p transitions in H-like ions such as O VIII or C VI all have the same oscillator strength.
}

of at least $10^{15} \mathrm{~cm}^{-2}$ is needed (Fig. 7 of Kinkhabwala et al. 2002), with some dependency on line broadening. Hence, if all observed Ly $\alpha$ emission is attributed to resonant scattering, the $\mathrm{H}$ column is greater than $\sim 10^{18} \mathrm{~cm}^{-2}$, assuming cosmic abundances. The significance of a discrete RRC emission feature rises as the column density of the scattering medium increases. In particular, the flux ratio of the RRC to Ly $\alpha$ rises from $\sim 0.1$ to 1.0 as the column density increases from $10^{16} \mathrm{~cm}^{-2}$ to $10^{18} \mathrm{~cm}^{-2}$. This is demonstrated using an atomic-state kinetics model in Figs. 1 and 2 of Behar et al. (2002). If we can estimate the luminosity and spectrum of the central source when it is obscured, we could get a rough idea of the covering fraction of the emitting gas.

For recombination or collisional line excitation the emission measure formalism can be applied to derive the product of density square and volume, $n_{\mathrm{e}}^{2} V$, and independent measurement of either quantity gives the other.

\subsection{Obscuration of the continuum in SSe}

Independent of the formation mechanisms of the emission lines, the decisive difference between SSa and SSe is the brightness of the continuum relative to the strengths of emission lines which are about the same in both subclasses. The most likely explanation for the weaker continuum emission in SSe is obscuration of the central source while some continuum emission can escape obscuration via Thomson scattering and reprocessing.

\subsubsection{Comparison to obscured and unobscured AGN}

Obscuration of the continuum with remaining predominant emission lines as in our SSe spectra has also been observed in some Active Galactic Nuclei (AGN). Indeed, this is the central concept of the unified model of AGN, in which the spectrum observed is strongly dependant on whether the accretion structures of the central supermassive black hole are observed at low or 


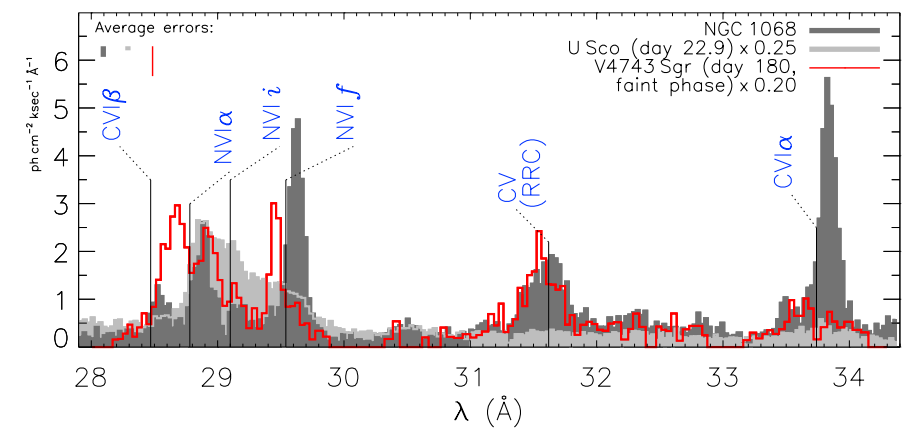

Fig. 9. Comparison of an XMM-Newton/RGS spectrum of AGN NGC 1068 (dark shades) with U Sco (light shades) and the last $5.5 \mathrm{ks}$ of the March 2003 Chandra observation of V4743 Sgr (thick red line; see Table 2). NGC 1068 contains strong radiative recombination features (RRC; Kinkhabwala et al. 2002). The C V RRC at $31.5 \AA$ can also be seen in V4743 Sgr but not in U Sco. (The concept is the same as described in Fig. 2.)

high inclination, leading to a view which is unobscured (type I) or obscured (type II) by a dusty torus at large radii respectively (Antonucci \& Miller 1985). For example, Kinkhabwala et al. (2002) and Brinkman et al. (2002) describe such an emission line spectrum from NGC 1068 and conclude that it is produced by photoionisation and photoexcitation, followed by recombination and de-excitation, respectively, either directly into the ground state or into lower excited states from where they cascade into the ground state. An AGN spectrum equivalent to an SSa is that of the unobscured AGN NGC 3783. Kaspi et al. (2002) report the detection of 135 absorption lines, 42 of which are unblended. In addition, they report line emission "filling in" the absorption, while the combination of absorption and emission lines is not interpreted as a P Cygni profiles. Their Fig. 1 around $19 \AA$ looks like the SSS spectrum of KT Eri around $24 \AA$ with one single emission line clearly detectable above the continuum (Fig. 2). In the case of NGC 3783, there are clear emission lines at longer wavelengths where there is no continuum which can be explained by photoexcitation by the high-energy continuum. On the other hand, emission lines belonging to transitions with energies higher than the Wien tail of an SSS continuum can not be photo-excited. Detailed analysis of a mixture of continuum emission and emission lines seen in NGC 1365 (Fig. 2 in Guainazzi et al. 2009) reveals a mixture of collisional and photo-ionisation.

Important features in the X-ray spectra of obscured AGN arise from RRC features which Guainazzi et al. (2009) attribute to a photoionised plasma. Their width depends on the mean electron velocity, or kinetic electron temperature. RRCs can only be observed in a plasma of electron temperatures up to some $100 \mathrm{eV}$, not sufficient to reach the ionisation stages of observed lines such as $\mathrm{O}$ VIII via collisional ionisation. The presence of narrow RRC features is a strong indicator for a cool plasma that reaches high ionisation stages via photoionisation.

The inverse process, absorption during ionisation, leads to absorption edges in the ionising continuum spectrum at the same energies as RRCs. In perfect ionisation equilibrium, neither RRC nor ionisation edges are observable. In obscured AGN, the ionisation source cannot be seen, and the recombination emission from the photoionised plasma is not balanced by the continuum emission with absorption edges from the source.

The only nova spectrum with RRC features was taken during the intermittent faint state in V4743 Sgr reported by Ness et al. (2003). In Fig. 9, we compare an XMM-Newton spectrum of the AGN NGC 1068 with U Sco and the faint spectrum of
V4743 Sgr with scaling factors applied as given in the legend. Apart from a scaling factor, the C V RRC feature at $31.5 \AA$ is identical in NGC 1068 and V4743 Sgr. The same width indicates similar electron temperatures. Also the strength in relation to the $\mathrm{N}$ VI lines is remarkably similar, however, the $\mathrm{N}$ VI lines are red shifted in NGC 1068 and blue shifted in V4743 Sgr while the $\mathrm{C} \mathrm{V} \mathrm{RRC} \mathrm{feature} \mathrm{resides} \mathrm{at} \mathrm{the} \mathrm{rest} \mathrm{wavelength.} \mathrm{The} \mathrm{spectrum} \mathrm{of}$ U Sco contains no RRC features (Orio et al. 2013), suggesting a different formation origin.

\subsubsection{Approximation of luminosity in SSa and SSe}

To illustrate the effect of continuum obscuration further, we compare the bolometric luminosities derived for SSa and SSe spectra. To avoid the ambiguity from the large parameter space of atmosphere models, we focus here on blackbody fits to illustrate the effects. Obtaining well constrained, credible parameters from atmosphere models is not the purpose of this project.

We use the parameters of the light blue blackbody curves included in Fig. 1, and in the top panel of Fig. 10, we show the values of $L_{\mathrm{bb}}$ versus $T_{\mathrm{bb}}$. It is well known that blackbody fits can yield unrealistic luminosities (Krautter et al. 1996) and consequently also temperatures. We use the values as indicators without giving error bars, as they are not the result of a full-physics modelling of the data. Atmosphere models have more flexibility to stay below the Eddington luminosity (see, e.g., Balman et al. 1998; Page et al. 2010), but there is no unique way of accomplishing that, and the multitude of possibilities may obscure the point we are aiming at. Major sources of uncertainty are the distance (values used are given in Table 2) and contamination by blending absorption and/or emission lines that complicate the determination of an appropriate model which we caution again was only done manually. In addition, sources with high values of $N_{\mathrm{H}}$ suffer from a high degree of systematic uncertainty as a large fraction of the radiation from the source is actually not visible to us and thus not available to constrain models that correspond to different values in bolometric luminosity and effective temperature.

The results for the new subclasses SSa and SSe are plotted with bullet and triangular symbols, respectively. In the top and right of the plot, distribution histograms are shown indicating that the distribution in temperature and luminosity yields higher values for SSa spectra. Clearly, the sample is too small to draw reliable conclusions, and more sources are desirable to populate the diagram.

In the bottom panel of Fig. 10, cumulative distributions are shown for the luminosities derived from SSe (blue) and SSa (red) that have more secure values. The distributions clearly show that systematically lower luminosities are derived for SSe spectra. A Wilcoxon rank sum test rejects the null hypothesis that both luminosity distributions agree on the $95 \%$ confidence level, thus supporting the conclusion that the central source is obscured in SSe compared to SSa.

\subsection{Effects from an accretion disc}

We found a systematic trend of high-inclination systems displaying SSe spectra with no confirmed case of an SSa spectrum emitted by a high-inclination system. The most obvious reason for obscuration of the central continuum in high-inclination systems is the accretion disc if present. The phenomenon of predominance of emission lines over weak continuum in highinclination systems has also been observed dwarf novae (DNe) in outburst (Hamilton et al. 2007), revealing similar conclusions 

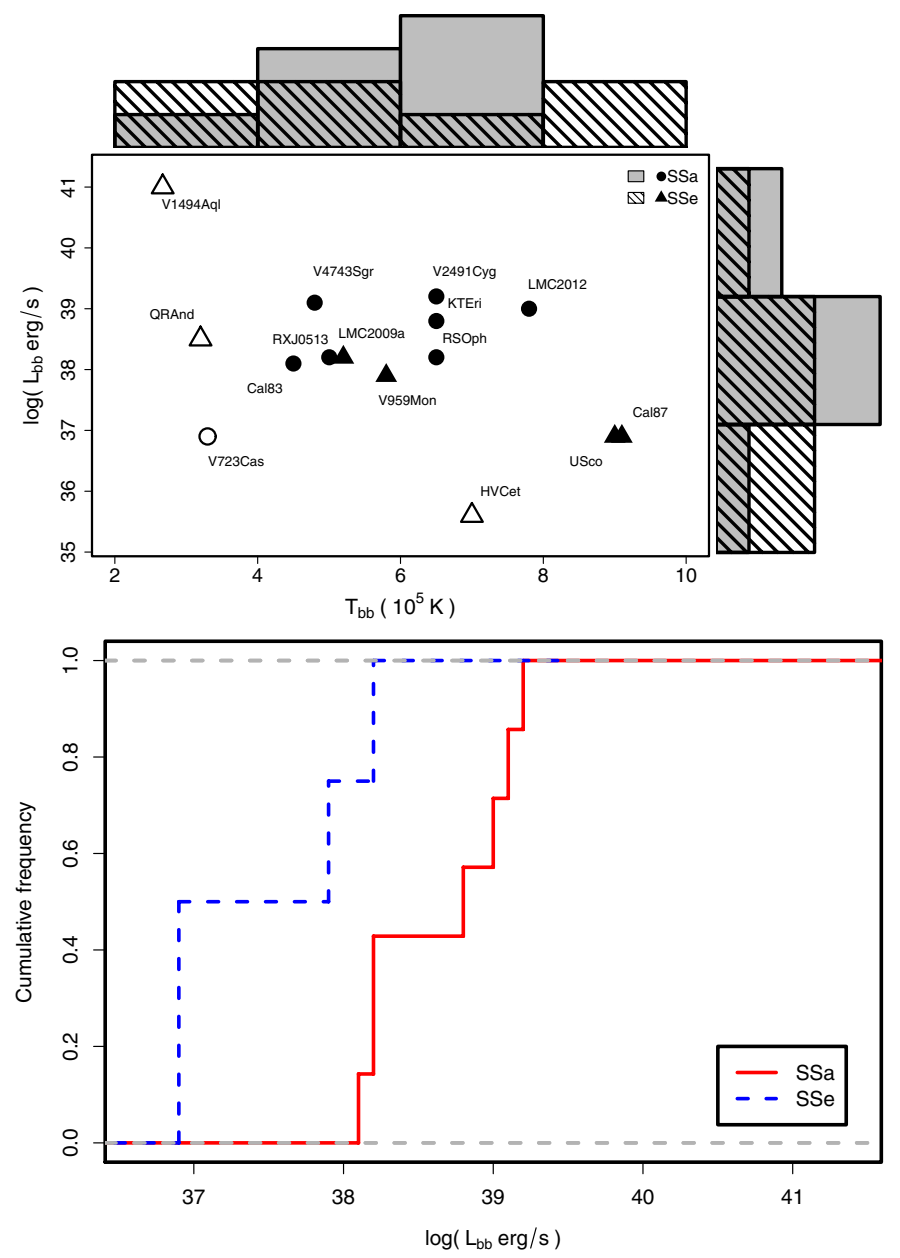

Fig. 10. Top: blackbody-estimated luminosities, $L_{\mathrm{bb}}$, versus temperature, $T_{\mathrm{bb}}$, determined from Planck functions fitted to the continuum component of SSa and SSe spectra, marked by bullet and triangular symbols, respectively. Open symbols indicate particularly high degree of uncertainty owed to line blends or high but poorly constrained amounts of photoelectric absorption. The hatched and shaded histograms in the top and right, decoded in the legend, give the number of SSe and SSa spectra in rough temperature and luminosity intervals. Bottom: cumulative distributions of derived luminosities for SSa and SSe, including only the systems with filled symbols in the top panel. SSa clearly yield higher luminosities. BB-derived luminosities and temperatures are indicative, as they are not the result of a full-physics modelling of the data, and for this reason error bars are not plotted.

drawn from the X-ray grating spectrum of U Sco (Ness et al. 2012a). Kuulkers et al. (2006) summarise that in DNe, eclipses affect the continuum more strongly than the lines which is consistent with our observation that SSe generally have relatively weaker continuum than SSa. Eclipsing DNe such as WZ Sge and OY Car are dominated more by emission lines than systems with lower inclination angle such as SS Cyg (Mauche 2004). Mauche \& Raymond (2000) found good spectral fits to EUVE spectra of OY Carinae using a model similar to obscured AGN (= Seyfert 2 galaxies) wherein the EUV radiation from the central regions and the disc is scattered into the line of sight by the system's photo-ionised disc wind. Similar processes were also observed in Low Mass X-ray Binaries (LMXB) which contain a neutron star that accretes via an accretion disc from a lowmass companion star. Kallman et al. (2003) presented X-ray grating spectra of the high-inclination LMXB 2S 0921-63 and also pointed out the analogous nature to obscured AGN as we do in Sect. 4.2.1.

To determine the limiting angle above which the central source is obscured by the accretion disc, we consider the ratio of the disc radius $R_{\mathrm{d}}$ and the outer disc edge $H_{\mathrm{d}}$ which can be estimated as a function of $M_{1}, M_{2}$ (primary and secondary masses, respectively) and orbital period. A standard thin $\alpha$-disc approximation (Frank et al. 1992) yields $H_{\mathrm{d}} / R_{\mathrm{d}} \sim 0.01$, thus $H_{\mathrm{d}}$ is small compared to $R_{\mathrm{d}}$ (and also to the white dwarf), allowing occultation only at extremely high inclination angles. However, there are additional processes that lead to a thicker outer edge of the disc such as the impact of the accretion stream from the secondary star (e.g., Frank et al. 1987; Armitage \& Livio 1998, invoked by Page et al. 2013) or the presence of the hot central SSS source irradiating the disc at a level of several $10^{5} \mathrm{~K}$ (Suleimanov et al. 1999, 2003). Accounting for the resulting "spray" at the outer disc edge, $H_{\mathrm{d}} / R_{\mathrm{d}}$ can reach values from 0.25 to 0.4 (see Fig. 3 in Schandl et al. 1997), thus yielding large disc heights compared to the radius of the central source. The spray can then eclipse the white dwarf also at lower inclination angle. From simple trigonometry, the arctangent of the $H / R$ ratio gives the angle between the disc plane and the white dwarf-spray line. It is the angle below which the white dwarf is obscured by the spray (assuming the white dwarf being a point source). The resulting inclination $\left(90^{\circ}-\right.$ this angle) is approximately $68^{\circ}$ for $H / R=0.4$ and $76^{\circ}$ for $H / R=0.25$. The central source can then be obscured by the spray above inclination, in the range from $68^{\circ}-76^{\circ 3}$. From trigonometric considerations, we find that for inclination angles below $68^{\circ}$, the white dwarf is unobscured, yielding an SSa spectrum, while above $76^{\circ}$, a pure SSe spectrum is expected, which is confirmed by Fig. 1. Between these two values, the white dwarf is partially eclipsed, yielding hybrid spectra between SSa and SSe. Since the spray is unlikely to be uniform in height above the disc, transitions between SSa and SSe may vary with the orbit, however, this can not be confirmed from the observations so far.

While an accretion disc is present in persistent $\mathrm{SSS}, \mathrm{CV}$ s in general, and in DNe, it might be absent in novae during their SSS phase. The initial explosion destroys the accretion disc. For example for USco, Drake \& Orlando (2010) performed hydrodynamic simulations of the initial blast concluding that the accretion disc was completely destroyed. For the novae in our sample, the disc may have either re-formed before our spectra were obtained, or it was never destroyed in the first place as proposed by, e.g., Walter \& Battisti (2011).

First signs of re-establishment of the accretion disc in U Sco were renewed optical flickering some 7 days after outburst (Worters et al. 2010; Munari et al. 2010). While a time scale of 7 days would be consistent with predictions of the mass loss rate from the companion by Hachisu et al. (2000), any renewed disc build-up needs to overcome the dynamic pressure of the radiatively driven outflow from the evolving central object at this early time (Drake \& Orlando 2010). Meanwhile, the X-ray studies during the later SSS phase have shown that an accretion disc may have reformed by the start of the SSS phase (Ness et al. 2012a). At a later time, the wind from the central object may have weakened. Furthermore, a disc may reform more rapidly after a nova explosion because of the heating effect on the secondary star that would cause enhanced Roche lobe overflow (e.g. van den Heuvel et al. 1992).

\footnotetext{
We note that, if the accretion disc is tilted or warped, the lower limit on the limiting angle can be even lower.
} 


\subsection{Other sources of $X$-ray variability}

The transitions between SSa and SSe were not seen during fainter episodes of RS Oph (see Fig. 5), and this system has in fact shown a correlation between brightness and hardness, lagging by 1000 s (Ness et al. 2007b; Schönrich \& Ness 2008). Associated changes in spectral shape suggest a physical origin such as photospheric expansion, leading to a decrease in brightness and shift of the SED to longer wavelengths at which the radiation is absorbed by the interstellar medium (Ness et al. 2007a).

Also, the decline in brightness in V4743 Sgr has been accompanied with a softening of the X-ray spectrum (Ness et al. 2003), and it is also the only nova showing RRC features during a faint state (Fig. 9). Recently, Rajoelimanana et al. (2013) have studied $\mathrm{X}$-ray on- and off states in Cal 83 which are inversely correlated with optical high- and low states. They found a softening of the $\mathrm{X}$-ray spectrum when the source increases in optical brightness. Perhaps the underlying processes to this type of variability are related to the high-amplitude variations frequently seen in novae during the early SSS phase. While the discussion of variability deserves close inspection, this is beyond the scope of this paper.

\section{Summary and conclusions}

Before XMM-Newton and Chandra revolutionised X-ray astronomy, the observed SSS spectra seen as plain blackbodies with no features in them that could be used to sensibly constrain any more sophisticated models. The X-ray gratings that can resolve lines are required to explore SSS spectra to deeper limits. These limits have not yet been reached by current-generation models, and we focus only on the data.

Over the last 13 years, high-resolution grating spectra of SSS and various types of novae during their SSS phase have been obtained. Here, we studied all available grating spectra and found remarkable similarities between some of them, even when taken from different systems at different times since outburst. While some SSS spectra exhibit emission from a photosphere with absorption lines, others are dominated by emission lines on top of a weak blackbody-like continuum. We define two SSS classes $\mathrm{SSa}$ and SSe for the former and latter types, respectively:

- The SSa class shows an X-ray spectrum of a blackbody-like continuum with clearly visible absorption lines. Emission lines may be present but are difficult to see.

- The SSe class shows an X-ray spectrum that is clearly dominated by emission lines from well-known species. They also contain a weak blackbody-like continuum of comparable intensities to the emission lines but without clear absorption lines.

We found two pairs of almost identical SSa spectra, RS Oph/V2491 Cyg and V4743 Sgr/KT Eri, and one pair of identical SSe spectra, U Sco/Cal 87. In addition, various intermediate spectra have been observed that contain characteristics of both.

All the evidence we gathered together points to an interpretation of obscuration of the central source in SSe spectra compared to SSa spectra:

- Systems with high inclination angles $>70^{\circ}$ are seen to have SSe spectra.

- In some novae, we find both SSa and SSe states, where the SSe state was always seen during episodes of reduced brightness, indicating obscuration of the continuum component.
- If the white dwarf is completely blocked, e.g. by the companion, blackbody-like continuum emission may still be observable because of Thomson scattering and reprocessing.

- We observed stronger emission lines in SSe at wavelengths where the underlying continuum is stronger, indicating photoexcitations as part of resonant line scattering.

- SSa spectra may contain emission lines of the same strengths as in SSe which are hidden between complex atmospheric features.

- Blackbody fits to the continuum component yield systematically lower luminosities in SSe than in SSa.

- Similar phenomena are known for other types of CVs, dwarf novae. Resemblance of an SSe spectrum with that of an obscured AGN supports an interpretation that central continuum emission is obscured in SSe.

Permanent obscuration in high-inclination angle systems implies the presence of an accretion disc. In nova systems, the disc may either have survived the initial explosion or it has reformed by the time of the start of the SSS phase.

We caution, however, that not all observed variability need be attributed to obscuration. The high amplitude oscillations in RS Oph described by Osborne et al. (2011) have not shown the characteristic fading to an SSe spectrum, but associated hardness changes. Likewise, recent studies of on- and off states in Cal 83 point to physical effects as hardness changes also occur during transitions between on- and off states. These effects are not the subject of this work but deserve closer inspection.

Acknowledgements. We thank the referee for a most inspiring and encouraging report. The authors appreciate useful discussions with M. Giustini about AGNs. We thank S. N. Shore who has helped with useful comments. This research has made use of data obtained with the gratings on board XMM-Newton and Chandra. XMM-Newton is an ESA science mission with instruments and contributions directly funded by ESA Member States and NASA. Software provided by the Chandra X-ray Center (CXC) in the application package CIAO was used to obtain science data. K. L. Page, J. P. Osborne, and A. P. Beardmore acknowledge financial support from the UK Space Agency. A. Dobrotka was supported by the Slovak Academy of Sciences Grant No. 1/0511/13 and by the ESA international fellowship. M. Henze acknowledges support from an ESA fellowship. V. A. R. M. Ribeiro acknowledges the South African SKA Project for funding the postdoctoral fellowship at the University of Cape Town. M. Hernanz acknowledges the Spanish grants AYA2011-24704 and 2009 SGR 315. S. Starrfield acknowledges partial support from NASA and NSF grants to ASU. E. Behar was supported by grant \#1163/10 from the Israel Science Foundation.

\section{References}

Alcock, C., Allsman, R. A., Alves, D., et al. 1997, MNRAS, 286, 483

Antonucci, R. R. J., \& Miller, J. S. 1985, ApJ, 297, 621

Armitage, P. J., \& Livio, M. 1998, ApJ, 493, 898

Balman, S., Krautter, J., \& Ögelman, H. 1998, ApJ, 499, 395

Barry, R. K., Mukai, K., Sokoloski, J. L., et al. 2008, in RS Ophiuchi (2006) and the Recurrent Nova Phenomenon, eds. A. Evans, M. F. Bode, T. J. O’Brien, \& M. J. Darnley, ASP Conf. Ser., 401, 52

Barsukova, E. A., \& Goranskii, V. P. 2003, Astron. Lett., 29, 195

Beardmore, A. P., Osborne, J. P., Page, K. L., et al. 2012, A\&A, 545, A116

Becker, C. M., Remillard, R. A., Rappaport, S. A., \& McClintock, J. E. 1998, ApJ, 506, 880

Behar, E., Kinkhabwala, A., Sako, M., et al. 2002, in Mass Outflow in Active Galactic Nuclei: New Perspectives, eds. D. M. Crenshaw, S. B. Kraemer, \& I. M. George, ASP Conf. Ser., 255, 43

Beuermann, K., Reinsch, K., Barwig, H., et al. 1995, A\&A, 294, L1

Bode, M. F. 1987, in RS Ophiuchi (1985) and the Recurrent Nova Phenomenon, ed. M. F. Bode, 241

Bos, M., Retter, A., McCormick, J., \& Velthuis, F. 2001, IAUcirc, 7610, 2

Brinkman, A. C., Kaastra, J. S., van der Meer, R. L. J., et al. 2002, A\&A, 396, 761

Burwitz, V., Starrfield, S., Krautter, J., \& Ness, J.-U. 2002a, in Classical Nova Explosions, eds. M. Hernanz, \& J. José, AIP Conf. Ser., 637, 377 
Burwitz, V., Starrfield, S., Krautter, J., \& Ness, J.-U. 2002b, in High Resolution X-ray Spectroscopy with XMM-Newton and Chandra, ed. G. Branduardi-Raymont

Burwitz, V., Reinsch, K., Greiner, J., et al. 2008, A\&A, 481, 193

Chesneau, O., Meilland, A., Banerjee, D. P. K., et al. 2011, A\&A, 534, L11

Crampton, D., Cowley, A. P., Hutchings, J. B., et al. 1987, ApJ, 321, 745

Darnley, M. J., Ribeiro, V. A. R. M., Bode, M. F., Hounsell, R. A., \& Williams, R. P. 2012, ApJ, 746, 61

Della Valle, M., Pasquini, L., Daou, D., \& Williams, R. E. 2002, A\&A, 390, 155

Dobrotka, A., Retter, A., \& Liu, A. 2008, A\&A, 478, 815

Dobrzycka, D., \& Kenyon, S. J. 1994, AJ, 108, 2259

Drake, J. J., \& Orlando, S. 2010, ApJ, 720, L195

Ebisawa, K., Mukai, K., Kotani, T., et al. 2001, ApJ, 550, 1007

Ebisawa, K., Rauch, T., \& Takei, D. 2010, AN, 331, 152

Frank, J., King, A. R., \& Lasota, J.-P. 1987, A\&A, 178, 137

Frank, J., King, A., \& Raine, D. 1992, Accretion power in astrophysics, Camb. Astrophys. Ser., 21

Goranskij, V. P., Katysheva, N. A., Kusakin, A. V., et al. 2007, Astrophys. Bull., 62,125

Greiner, J. 1996, Lect. Notes Phys., 472, Supersoft X-Ray Sources (Berlin: Springer Verlag)

Greiner, J., Hasinger, G., \& Kahabka, P. 1991, A\&A, 246, L17

Greiner, J., Iyudin, A., Jimenez-Garate, M., et al. 2004, in Rev. Mex. Astron. Astrofis. Conf. Ser. 20, eds. G. Tovmassian, \& E. Sion, 18

Guainazzi, M., Risaliti, G., Nucita, A., et al. 2009, A\&A, 505, 589

Hachisu, I., Kato, M., Kato, T., Matsumoto, K., \& Nomoto, K. 2000, ApJ, 534, L189

Hachisu, I., Kato, M., \& Kato, T. 2004, ApJ, 606, L139

Hamilton, R. T., Urban, J. A., Sion, E. M., et al. 2007, ApJ, 667, 1139

Hartmann, H. W., \& Heise, J. 1996, in Supersoft X-Ray Sources, ed. J. Greiner (Berlin: Springer Verlag), Lect. Notes Phys., 472, 25

Hartmann, H. W., \& Heise, J. 1997, A\&A, 322, 591

Hauschildt, P. H., Wehrse, R., Starrfield, S., \& Shaviv, G. 1992, ApJ, 393, 307

Helton, L. A., Woodward, C. E., Vanlandingham, K., \& Schwarz, G. J. 2008, CBET, 1379, 1

Hounsell, R., Bode, M. F., Hick, P. P., et al. 2010, ApJ, 724, 480

Hutchings, J. B., Winter, K., Cowley, A. P., Schmidtke, P. C., \& Crampton, D. 2002, AJ, 124, 2833

Iijima, T., \& Esenoglu, H. H. 2003, A\&A, 404, 997

Kahabka, P. 1996, A\&A, 306, 795

Kahabka, P. 1997, in IAU Colloq. 163, Accretion Phenomena and Related Outflows, eds. D. T. Wickramasinghe, G. V. Bicknell, \& L. Ferrario, ASP Conf. Ser., 121, 730

Kahabka, P., \& van den Heuvel, E. P. J. 1997, ARA\&A, 35, 69

Kahabka, P., Pietsch, W., \& Hasinger, G. 1994, A\&A, 288, 538

Kallman, T. R., Angelini, L., Boroson, B., \& Cottam, J. 2003, ApJ, 583, 861

Kaspi, S., Brandt, W. N., George, I. M., et al. 2002, ApJ, 574, 643

Kinkhabwala, A., Sako, M., Behar, E., et al. 2002, ApJ, 575, 732

Krautter, J., Ögelman, H., Starrfield, S., Wichmann, R., \& Pfeffermann, E. 1996, ApJ, 456, 788

Kuulkers, E., Norton, A., Schwope, A., \& Warner, B. 2006, in X-rays from cataclysmic variables, eds. W. H. G. Lewin, \& M. van der Klis (Cambridge University Press), 421

Lanz, T., Telis, G. A., Audard, M., et al. 2005, ApJ, 619, 517

Long, K. S., Helfand, D. J., \& Grabelsky, D. A. 1981, ApJ, 248, 925

Lyke, J. E., \& Campbell, R. D. 2009, AJ, 138, 1090

Macri, L. M., Stanek, K. Z., Bersier, D., Greenhill, L. J., \& Reid, M. J. 2006, ApJ, 652, 1133

Mauche, C. W. 2004, Rev. Mex. Astron. Astrofis. 20, 174

Mauche, C. W., \& Raymond, J. C. 2000, ApJ, 541, 924

McGowan, K. E., Charles, P. A., Blustin, A. J., et al. 2005, MNRAS, 364, 462

Morgan, G. E., Ringwald, F. A., \& Prigge, J. W. 2003, MNRAS, 344, 521

Mukai, K., \& Ishida, M. 2001, ApJ, 551, 1024

Munari, U., Dallaporta, S., \& Castellani, F. 2010, Inf. Bull. Variable Stars, 5930, 1

Munari, U., Dallaporta, S., Castellani, F., et al. 2013, MNRAS, 435, 771

Nelson, T., Orio, M., Cassinelli, J. P., et al. 2008, ApJ, 673, 1067

Ness, J. 2010, Astron. Nachr., 331, 179

Ness, J. U. 2012, Bull. Astron. Soc. India, 40, 353
Ness, J., Starrfield, S., Burwitz, V., et al. 2003, ApJ, 594, L127

Ness, J., Starrfield, S., Jordan, C., Krautter, J., \& Schmitt, J. H. M. M. 2005 , MNRAS, 364, 1015

Ness, J., Schwarz, G. J., Retter, A., et al. 2007a, ApJ, 663, 505

Ness, J., Starrfield, S., Beardmore, A., et al. 2007b, ApJ, 665, 1334

Ness, J., Drake, J. J., Beardmore, A. P., et al. 2009a, AJ, 137, 4160

Ness, J., Drake, J. J., Starrfield, S., et al. 2009b, AJ, 137, 3414

Ness, J., Osborne, J. P., Dobrotka, A., et al. 2011, ApJ, 733, 70

Ness, J., Schaefer, B. E., Dobrotka, A., et al. 2012a, ApJ, 745, 43

Ness, J.-U., Shore, S. N., Drake, J. J., et al. 2012b, ATel, 4569

Orio, M., Parmar, A., Benjamin, R., et al. 2001, MNRAS, 326, L13

Orio, M., Parmar, A. N., Greiner, J., et al. 2002, MNRAS, 333, L11

Orio, M., Behar, E., Gallagher, J., et al. 2013, MNRAS, 429, 1342

Osborne, J. P., Page, K. L., Beardmore, A. P., et al. 2011, ApJ, 727, 124

Page, K. L., Osborne, J. P., Evans, P. A., et al. 2010, MNRAS, 401, 121

Page, K. L., Osborne, J. P., Wagner, R. M., et al. 2013, ApJ, 768, L26

Parmar, A. N., Kahabka, P., Hartmann, H. W., et al. 1997, A\&A, 323, L33

Parmar, A. N., Kahabka, P., Hartmann, H. W., Heise, J., \& Taylor, B. G. 1998, A\&A, 332, 199

Ragan, E., Brozek, T., Suchomska, K., et al. 2009, ATel, 2327

Rajoelimanana, A. F., Charles, P. A., Meintjes, P. J., Odendaal, A., \& Udalski, A. 2013, MNRAS, 432, 2886

Rauch, T., Orio, M., Gonzales-Riestra, R., et al. 2010, ApJ, 717, 363

Ribeiro, V. A. R. M., Bode, M. F., Darnley, M. J., et al. 2009, ApJ, 703, 1955

Ribeiro, V. A. R. M., Darnley, M. J., Bode, M. F., et al. 2011, MNRAS, 412, 1701

Ribeiro, V. A. R. M., Bode, M. F., Darnley, M. J., et al. 2013a, MNRAS, 433, 1991

Ribeiro, V. A. R. M., Munari, U., \& Valisa, P. 2013b, ApJ, 768, 49

Rohrbach, J. G., Ness, J., \& Starrfield, S. 2009, AJ, 137, 4627

Sala, G., Hernanz, M., Ferri, C., \& Greiner, J. 2008, ApJ, 675, L93

Sala, G., Hernanz, M., Ferri, C., \& Greiner, J. 2010, Astron. Nachr., 331, 201

Schaefer, B. E. 1990, ApJ, 355, L39

Schaefer, B. E. 2010, ApJS, 187, 275

Schaeidt, S., Hasinger, G., \& Truemper, J. 1993, A\&A, 270, L9

Schandl, S., Meyer-Hofmeister, E., \& Meyer, F. 1997, A\&A, 318, 73

Schlegel, E. M., Schaefer, B., Pagnotta, A., et al. 2010, ATel, 2419

Schmidtke, P. C., \& Cowley, A. P. 2006, AJ, 131, 600

Schönrich, R. A., \& Ness, J.-U. 2008, in RS Ophiuchi (2006) and the Recurrent Nova Phenomenon, eds. A. Evans, M. F. Bode, T. J. O'Brien, \& M. J. Darnley, ASP Conf. Ser., 401, 291

Schwarz, G. J., Ness, J.-U., Osborne, J. P., et al. 2008, ATel, 1847

Schwarz, G. J., Ness, J., Osborne, J. P., et al. 2011, ApJS, 197, 31

Shore, S. N., Schwarz, G., Bond, H. E., et al. 2003, AJ, 125, 1507

Shore, S. N., Augusteijn, T., Ederoclite, A., \& Uthas, H. 2011, A\&A, 533, L8

Sokoloski, J. L., Crotts, A. P. S., Lawrence, S., \& Uthas, H. 2013, ApJ, 770, L33

Southwell, K. A., Livio, M., Charles, P. A., O’Donoghue, D., \& Sutherland, W. J. 1996, ApJ, 470, 1065

Starrfield, S., Iliadis, C., \& Hix, W. R. 2008, in Classical Novae, eds. M. Bode, \& A. Evans (Cambridge University Press), 77

Suleimanov, V., Meyer, F., \& Meyer-Hofmeister, E. 1999, A\&A, 350, 63

Suleimanov, V., Meyer, F., \& Meyer-Hofmeister, E. 2003, A\&A, 401, 1009

Takei, D., Drake, J. J., Ness, J.-U., et al. 2012, ATel, 4116

Thoroughgood, T. D., Dhillon, V. S., Littlefair, S. P., Marsh, T. R., \& Smith, D. A. 2001, MNRAS, 327, 1323

Tofflemire, B., Orio, M., Kuulkers, E., et al. 2011, ATel, 3762

Tomov, T., Munari, U., Kolev, D., Tomasella, L., \& Rejkuba, M. 1998, A\&A, 333, L67

Truemper, J. 1992, QJRAS, 33, 165

Uthas, H., Knigge, C., \& Steeghs, D. 2010, MNRAS, 409, 237

van den Heuvel, E. P. J., Bhattacharya, D., Nomoto, K., \& Rappaport, S. A. 1992, A\&A, 262, 97

van Rossum, D. R. 2012, ApJ, 756, 43

Vanlandingham, K. M., Schwarz, G., Starrfield, S., et al. 2007, in BAAS 39, Am. Astron. Soc. Meeting Abst. \#210, 99

Walter, F. M., \& Battisti, A. 2011, in BAAS, 43, Am. Astron. Soc. Meeting Abst. \#217, \#338.11

Worters, H. L., Eyres, S. P. S., Rushton, M. T., \& Schaefer, B. 2010, IAUC, 9114 